\title{
Actitudes y creencias hacia las matemáticas: un estudio comparativo entre estudiantes y profesores
}

Beliefs and attitudes toward mathematics: a comparative study between students and teachers

\author{
Volumen 17, Número 1 \\ Enero-Abril \\ pp. 1-45
}

Este número se publicó el $1^{\circ}$ de enero de 2017

DOI: http://dx.doi.org/10.15517/aie.v17i1.27473

Ronny Gamboa Araya
Tania Elena Moreira-Mora

Revista indizada en REDALYC, $\underline{\text { SCIELO }}$

Revista distribuida en las bases de datos:

\section{LATINDEX, DOAJ, REDIB, IRESIE, CLASE, DIALNET, SHERPA/ROMEO, QUALIS-CAPES, MIAR}

Revista registrada en los directorios:

ULRICH'S, $\underline{\text { REDIEE}}, \underline{\text { RINACE}}, \underline{\text { OEI }}$ MAESTROTECA, PREAL, CLACSO 


\title{
Actitudes y creencias hacia las matemáticas: un estudio comparativo entre estudiantes y profesores
}

\author{
Beliefs and attitudes toward mathematics: a comparative study between students and
} teachers

\section{Ronny Gamboa Araya ${ }^{1}$ Tania Elena Moreira-Mora ${ }^{2}$}

\begin{abstract}
Resumen: La enseñanza y el aprendizaje de las matemáticas se han asociado con bajos resultados académicos, lo que ha creado un ambiente negativo en torno a esta disciplina. Algunas investigaciones han señalado que las creencias y actitudes hacia las matemáticas, por parte de los estudiantes, podrían explicar, en parte, el origen de la problemática que existe en dicha materia. También, han destacado la influencia que el docente posee en la formación de estos componentes del dominio afectivo de sus alumnos. Este artículo muestra los resultados de una investigación en la cual el objetivo fue analizar las actitudes y creencias hacia la disciplina por parte de estudiantes y docentes. La investigación se llevó a cabo en décimo año, con sus respectivos docentes de matemáticas, y participaron tres instituciones de secundaria de Costa Rica. Para el logro de los objetivos, se utilizó un diseño mixto; la recolección de datos se realizó mediante encuestas, observación, entrevistas y un grupo focal. Los resultados obtenidos muestran coincidencias y divergencias para ambos grupos de participantes. Estudiantes y docentes coinciden en señalar que para aprender matemáticas es necesario, entre otros aspectos, esfuerzo, dedicación y sentir gusto por la materia. Valoran la aplicabilidad de la disciplina, aunque esto no se utiliza como un recurso didáctico en las lecciones. El estudiantado, en general, no presenta interés en el aprendizaje de las matemáticas; mientras que el profesorado las valora como una disciplina agradable y fundamental en la formación de toda persona. Lo anterior genera un reto para el docente, quien debe innovar en su labor de aula para incidir, positivamente, en el domino afectivo de sus estudiantes.
\end{abstract}

Palabras clave: actitudes, creencias, matemáticas, estudiantes, profesores.

Abstract: The teaching and learning of Mathematics have been associated with low academic performance, which has created a negative atmosphere around it. Some research has indicated that beliefs and attitudes towards Mathematics, by students, may partly explain the origin of this problem. They also highlighted the influence that the teacher has in the formation of these components of the affective domain of their students. This article shows the results of an investigation where the objective was to analyze the attitudes and beliefs of students and teachers toward this discipline. The research was carried out in three secondary institutions in Costa Rica with tenth-grade students and their mathematics teachers. To achieve the goals, a mixed design research type was used; data collection was conducted through questionnaires, observation, interviews, and a focus group. The results show similarities and differences for both groups of participants. Students and teachers agree that, to learn Mathematics, is necessary, among other things, effort, dedication and feel like for the discipline. They value the applicability of discipline, although this is not used as a teaching resource in the lessons. Students, in general, have no interest in learning mathematics; while mathematics teachers value it as a likable and fundamental in the education of each person. This creates a challenge for teachers, which must innovate in their classroom work to influence positively in the affective domain of their students.

Keywords: attitudes, beliefs, mathematics, students, teachers.

${ }^{1}$ Doctor en Educación de la Universidad Estatal a Distancia de Costa Rica. Máster en Matemática Educativa por el CINVESTAV. Investigador y académico de la Escuela de Matemática de la Universidad Nacional de Costa Rica. Correo electrónico rgamboa@una.cr

${ }^{2}$ Doctora en Educación de la Universidad Estatal a Distancia de Costa Rica. Magíster en Evaluación Educativa. Investigadora y Profesional en Asesoría Estudiantil del Instituto Tecnológico de Costa Rica. Correo electrónico tmoreira@itcr.ac.cr

Artículo recibido: 5 de abril, 2016

Enviado a corrección: 27 de junio, 2016

Aprobado: 5 de diciembre, 2016 


\section{Introducción}

La enseñanza y aprendizaje de las matemáticas se ha asociado, comúnmente, con bajos resultados, fracasos académicos, obstáculos cognitivos y una serie de problemas que han creado un ambiente negativo en torno a esta disciplina.

En los últimos 25 años, la preocupación de distintos investigadores sobre la competencia matemática de los estudiantes ha hecho converger en cinco categorías las aptitudes que estos deberían adquirir para tener una buena disposición en este tema: conocimiento matemático, métodos heurísticos, metaconocimientos, habilidades de autorregulación y actitudes y creencias positivas sobre las matemáticas y su aprendizaje. (Gómez-Chacón, 2007)

Este último aspecto ha adquirido trascendencia debido a la relación que las actitudes y creencias sobre las matemáticas poseen con la enseñanza y aprendizaje de la disciplina. Por una parte, en este sentido, Eleftherios y Theodosios (2007) apuntan que la dificultad de esta se correlaciona con la creencia sobre su poca utilidad, con la aversión hacia la materia, el bajo rendimiento y la poca capacidad matemática. Por otra parte, "el amor por las matemáticas" se correlaciona positivamente con el alto rendimiento y capacidad matemática.

El importante papel de las creencias, el afecto y la motivación en el aprendizaje de la disciplina es bien reconocido en la educación matemática, pues las creencias de los estudiantes sobre la disciplina y las actitudes que poseen tienen un impacto en su compromiso, rendimiento, comportamiento y desempeño en la disciplina, especialmente en la resolución de problemas. (Eleftherios y Theodosios, 2007; Vieytes, 2009; Briley, Thompson e Iran-Nejad, 2009; Hassi y Laursen, 2009; Suthar y Tarmizi, 2010; Hekimoglu y Kittrell, 2010; Akay y Boz, 2010; Stramel, 2010)

La mejor manera de explicar cómo los estados emocionales influyen en el rendimiento es partir de los pensamientos, las actitudes y las creencias que determinan los sentimientos y las emociones. "Es decir, no son los hechos reales, sino el significado y las evaluaciones que el sujeto realiza las que producen cambios en las emociones y en los estados de ánimo" (Candia, 2009, p.2). Por ello, surge la necesidad de analizar las actitudes y creencias hacia las matemáticas del estudiantado y profesorado con el propósito de encontrar explicaciones, y efectuar comparaciones, sobre este fenómeno, que sirvan de sustento para la toma de decisiones con respecto a eventuales líneas de capacitación docente y para la evaluación de la práctica educativa de su aula, tendiente a mejorar, desde ahí, el factor emocional del alumnado. 
En este sentido, la investigación se enfocó en determinar las actitudes y creencias hacia las matemáticas del grupo estudiantil de décimo año y de los profesores de la disciplina en tres colegios académicos públicos diurnos de Costa Rica, para luego analizarlas. En las secciones posteriores se profundiza en aspectos teóricos, el diseño y los instrumentos de recolección de datos utilizados para el logro de los objetivos anteriores.

\section{Referente teórico}

\subsection{Actitudes hacia las matemáticas}

Las actitudes hacia las matemáticas se entienden como la predisposición evaluativa del estudiante que establece las intenciones personales hacia la disciplina e influyen en su comportamiento como aprendiz (Gil, Blanco y Guerrero, 2005). Según Martínez (2008), tienen que ver con la valoración, atracción, aprecio, satisfacción, curiosidad y el interés por la disciplina y su aprendizaje, acentuando más el componente afectivo que el cognitivo, y se caracterizan por considerar las capacidades de los sujetos y su modo de utilizarlas.

De acuerdo con Abraham et al., (2010) y Sánchez, Becerra, García y Contreras (2010), las actitudes están constituidas por tres componentes:

- El componente cognitivo, que incluye el dominio de hechos, opiniones, pensamientos, valores, conocimientos, expectativas, ideas y percepciones sobre el objeto de la actitud.

- El componente afectivo, que corresponde a los sentimientos que el sujeto tiene y la intensidad de estos, en este caso hacia las matemáticas. Incluye la aceptación (percepción del individuo sobre las matemáticas), motivación (impulso que conduce a una persona a elegir y realizar una acción), interés (expectativa con respecto a su relación con las matemáticas), bloqueo emocional (son fobias o inseguridades que limitan al individuo y no le permiten estar abierto a situaciones y estímulos que le pueden resultar gratificantes).

- El componente conductual, dado por la respuesta que el sujeto tiene en reacción al objeto de la actitud, la cual podría ser de rechazo, confianza, compañerismo, curiosidad, entre otros.

La actitud se acompaña de creencias y justificaciones que funcionan como un sistema de explicación, por lo que la opinión de los estudiantes, basada en sus creencias, desencadena actitudes hacia las matemáticas que condicionan su forma de actuar (Naranjo y Segura, 2010). 
El aula es el primer y más importante agente moldeador de las actitudes de los estudiantes, y dentro del aula, cobra especial significación la actuación del docente, en la formación y cambio de las actitudes de sus alumnos. El docente se convierte así, en un modelo a seguir, en un poderoso reforzador y promotor de actitudes positivas en el aula de clases. Desde esta perspectiva, las conductas asumidas por el docente, en gran medida tendrán un efecto transformador para sus alumnos. (Álvarez, 2007, p.89)

Aunado a lo anterior, Naranjo (2010) indica que las actitudes se adquieren de forma directa e indirecta. El modo directo es cuando la persona las adquiere mediante la experiencia, lo cual le produce satisfacción y significado; por su parte, en el modo indirecto, el individuo adquiere la actitud por medio de otros, a quienes respeta y admira, y que se convierten en modelos auténticos.

Las actitudes de los estudiantes hacia las matemáticas están determinadas por sus características particulares, las cuales están relacionadas con su autoimagen académica y la motivación de logro; para que un estudiante tenga éxito en matemáticas es necesario, pero no suficiente, que él tenga un concepto positivo de sí mismo, de su competencia para el trabajo escolar y que las actividades que desarrolla le provean de información que lo hagan sentirse competente y capaz, lo que contribuirá a consolidar un autoconcepto positivo. (Gil, Blanco y Guerrero, 2006)

\subsection{Creencias hacia las matemáticas}

Las creencias hacia las matemáticas se entienden como el conjunto de conocimientos, valores, ideologías, necesidades psicológicas, deseos, metas, entre otras, que posee una persona acerca de esta y su enseñanza (Op't Eynde, De Corte y Verschaffel, 2002; Parra, 2005), las cuales según Op't Eynde et al. (2002) se clasifican en las siguientes categorías y subcategorías:

- Creencias sobre la educación matemática, que incluyen aquellas relacionadas con las matemáticas, las cuales, según Ernest (1989), se pueden basar en una visión instrumentalista (considera que las matemáticas constituyen una acumulación de hechos, reglas y habilidades que pueden ser usadas en la ejecución de algún fin externo), platónica (concibe que las matemáticas son un cuerpo de conocimientos estático y unificado; son descubiertas, no creadas) o de resolución de problemas (visualiza las matemáticas como un campo de creación e invención humana en 
continua expansión, que son un producto cultural no acabado y sus resultados están abiertos a la revisión).

- Creencias de los individuos sobre sí mismos, las cuales se relacionan con su autoimagen con respecto a la disciplina y al proceso de aprendizaje de las matemáticas. Con respecto al proceso de aprendizaje de la disciplina, Solé (1999) indica que los estudiantes durante este, además de construir significados de los contenidos de la enseñanza, realizan representaciones de las situaciones de aprendizaje que podrían percibirse como un reto estimulante o inaccesible e inabordable según sus posibilidades. Sin embargo, a la vez, señala la autora, realizan representaciones sobre sí mismos como personas competentes, interesantes, capacitados para resolver problemas, o todo lo contrario.

- Creencias de los individuos sobre su contexto, entre las que se pueden distinguir aquellas relacionadas con el papel y el funcionamiento del profesor; incluyen las creencias asociadas a su tendencia didáctica, ya sea tradicionalista, tecnológica, espontaneísta e investigativa (Contreras, 1998); imagen de un buen profesor de matemáticas y la percepción sobre el docente de la disciplina.

Sobre este aspecto, Contreras (1998) plantea que, según ciertas características del docente y su práctica educativa, existen cuatro tendencias didácticas que rigen esta: tradicionalista, que se caracteriza por el uso de la exposición magistral y el libro de texto como material curricular; tecnológica, en la cual el profesor no expone los contenidos en su fase final, sino que simula su proceso de construcción, apoyándose en estrategias expositivas; espontaneísta, la cual se caracteriza por que el docente propone actividades de manipulación de modelos, a través de las cuales se espera que se produzca un conocimiento no organizado; investigativa, en la cual el profesor organiza el proceso que guiará al estudiante a la adquisición de los conocimientos determinados a través de su investigación.

Los anteriores aspectos teóricos relacionados con las creencias y actitudes hacia las matemáticas se utilizaron como base para el diseño de la investigación y el análisis de los datos. Los aspectos metodológicos se presentan en el siguiente apartado.

\section{Metodología}

La investigación se basó en un diseño mixto de dos etapas o fases: la primera fue cuantitativa y la segunda cualitativa. En la primera, se identificó las actitudes y creencias del 
estudiantado y docentes con respecto a las matemáticas. En la segunda, se profundizó en los significados e interpretaciones de esas percepciones en los participantes.

Para el logro de los objetivos propuestos, según la fase cuantitativa, se confeccionó dos cuestionarios. Para ello se tomaron como base los supuestos teóricos relacionados con las actitudes y creencias hacia las matemáticas y los instrumentos aplicados en otras investigaciones. Consistió en una escala tipo Likert con cinco valores escalares (muy de acuerdo, de acuerdo, ni de acuerdo, ni en desacuerdo, en desacuerdo, muy en desacuerdo).

El dirigido a estudiantes constaba de 127 preguntas, 5 de ellas correspondían a información general, 47 con actitudes hacia la materia y 75 relacionadas con las creencias hacia la disciplina. Para el caso de los docentes, el cuestionario estaba conformado por 122 ítems, distribuidos en 7, 40 y 75, respectivamente.

Las preguntas para estudiantes y docentes eran similares; algunas solo fueron adecuadas al rol de cada uno. Sin embargo, muchas poseían la misma redacción con el propósito de realizar comparaciones entre las respuestas. Las preguntas se plantearon referidas a las actitudes hacia las matemáticas según sus componentes: componente cognitivo, las preguntas se relacionaban con la percepción que tiene el individuo de sí mismo en su relación con las matemáticas, percepciones referidas a las matemáticas e ideas acerca de las situaciones escolares en las que el individuo se relaciona con las matemáticas; componentes afectivos, los ítems se relacionaban con aspectos como la aceptación, motivación, interés y expectativas con respecto a las matemáticas, así como con fobias o inseguridades que limitan al individuo y no le permiten estar abierto a situaciones y estímulos que le pueden resultar gratificantes; componentes conductuales, las preguntas se relacionaban con acciones de rechazo, confianza o curiosidad con respecto a las matemáticas.

En el caso de las creencias hacia la disciplina, se relacionaban con las creencias sobre las matemáticas (instrumentalista, platónica y resolución de problemas), creencias de los individuos con respecto a sí mismos en matemáticas y creencias asociadas al papel del profesor dentro del contexto de la clase (sobre su tendencia didáctica: tradicionalista, tecnológica, espontaneísta, investigativa; imagen de un buen profesor de matemáticas y percepción del docente). Para profundizar en estas concepciones, pueden referirse a Contreras. $(1998,2009)$

Para obtener evidencias de validez de contenido de los instrumentos, se recurrió a la consulta constante de la teoría relacionada con el tema de investigación, en conjunción con el criterio de jueces. Para ello, se contó con la participación de cinco docentes en el área de 
educación matemática. A cada uno de ellos se le envió un correo electrónico en el cual se le invitaba a participar y al cual respondió positivamente. Posteriormente, se le envió la versión inicial de los cuestionarios para estudiantes y docentes para que evaluaran los ítems según los siguientes criterios: no es pertinente, excluir del instrumento; parcialmente pertinente, mantener en el instrumento pero con modificaciones; totalmente pertinente, incluir en el instrumento sin modificaciones.

Para la evaluación respectiva, se confeccionaron dos instrumentos, uno para cada cuestionario, en el cual a los jueces se les presentaban tanto los problemas y subproblemas de investigación como los objetivos del estudio. Además, se adjuntaron dos cuadros donde se mostraban los insumos tomados en cuenta para la confección de estos.

Una vez realizadas las modificaciones respectivas producto del proceso anterior, se llevó a cabo una prueba piloto con la participación de 55 estudiantes de décimo de la provincia de Heredia de Costa Rica y 19 docentes que estuvieran impartiendo o hubiesen impartido dicho nivel. Ninguno de los participantes pertenecía a la muestra de la investigación. Una vez aplicados los cuestionarios, se procedió a la digitación de los datos en el SPSS y se realizó el análisis del alfa de Cronbach para cada uno de los componentes de las actitudes hacia las matemáticas y cada una de las dimensiones en las que se clasificó las creencias hacia la disciplina. Los resultados obtenidos se pueden observar en la tabla 1.

Tabla No 1. Medidas de confiabilidad de los componentes del cuestionario sobre actitudes y creencias hacia las matemáticas

\begin{tabular}{|c|c|c|}
\hline Componente o dimensión & Estudiantes & Docentes \\
\hline Componente cognitivo & 0,900 & 0,820 \\
\hline Componente afectivo & 0,901 & 0,857 \\
\hline Componente conductual & 0,883 & 0,842 \\
\hline Actitudes hacia las matemáticas & 0,960 & 0,829 \\
\hline Creencia sobre las matemáticas & 0,724 & 0,805 \\
\hline $\begin{array}{l}\text { Creencias sobre los individuos con respecto a sí mismos en } \\
\text { matemáticas }\end{array}$ & 0,845 & 0,698 \\
\hline $\begin{array}{l}\text { Creencias sobre el papel del profesor dentro del contexto de la } \\
\text { clase }\end{array}$ & 0,923 & 0,758 \\
\hline Creencias hacia las matemáticas & 0,921 & 0,810 \\
\hline
\end{tabular}

Fuente: Elaboración propia. 
Una vez efectuado el análisis anterior, se realizó un estudio por componentes principales, con el propósito de evaluar si cada uno de los factores o categorías establecidas estaban representados por los ítems propuestos y que cada ítem estuviera asociado a un solo factor o categoría, lo anterior con el objetivo de depurar las escalas e identificar los ítems multidimensionales y con baja discriminación. Para la recolección de los datos en la fase cualitativa, se utilizó observación no participante, entrevistas y un grupo focal.

\subsection{Participantes}

En la fase cuantitativa de la investigación participó el estudiantado de décimo año de tres instituciones académicas públicas diurnas de la región educativa de la provincia de Heredia de Costa Rica. Se seleccionaron estas instituciones debido a que la cercanía de ellas permitió trabajar con una población con características similares, de fácil acceso para los investigadores.

En cada una de las instituciones, se trabajó con todas las secciones de décimo año de estos colegios, por lo que no se utilizó ninguna muestra. En total fueron 506 estudiantes y cuatro docentes. Es importante señalar que, debido al poco número de docentes, los datos obtenidos por el cuestionario se analizaron únicamente desde un enfoque descriptivo.

Para la fase cualitativa se escogió una sección, en dos instituciones, para realizar una observación no participante durante, al menos, tres meses. De todas las instituciones se eligió a cuatro alumnos, según el criterio del profesor respectivo y de acuerdo con los rasgos requeridos, para indagar sobre las actitudes y creencias hacia la disciplina por parte de ellos. En total, se entrevistaron doce estudiantes y las dos docentes de los grupos objeto de observación. Dado que en una institución no fue posible realizar una observación no participante, se escogió a seis estudiantes para realizar un grupo focal e indagar en sus actitudes y creencias hacia la disciplina.

\section{Resultados}

Los resultados que se muestran a continuación representan una síntesis de los datos obtenidos al finalizar las dos fases de la investigación. Para el análisis descriptivo de los datos obtenidos por medio de la aplicación de los instrumentos, se realizaron las siguiente agrupaciones en las opciones: Muy de acuerdo y De acuerdo en De acuerdo y Muy en desacuerdo y En desacuerdo en En desacuerdo; la opción Ni de acuerdo ni en desacuerdo 
se etiquetó como neutral. Esto con el fin de simplificar la presentación de la información en las tablas, sin perder el sentido de las respuestas por parte de los participantes.

\subsection{Actitudes hacia las matemáticas}

El análisis de las actitudes hacia las matemáticas, de estudiantes y docentes, se presenta según cada uno de los componentes de estas, indicados en el apartado 2.

\subsubsection{Actitudes de los estudiantes hacia las matemáticas}

\subsubsection{Componente cognitivo}

Los resultados se muestran en la tabla 2. Los estudiantes encuestados, en su mayoría, señalan confiar en ellos cuando tienen que resolver un problema de matemáticas. Aunado a esto, un porcentaje importante indica que se considera capaz o hábil en la materia y que puede comprender el material impreso que se usa en la clase.

Tabla No 2. Porcentaje de respuestas dado por los estudiantes encuestados para los ítems asociados con el componente cognitivo

Ítem $\begin{gathered}\text { De } \\ \text { acuerdo }\end{gathered} \quad$ Neutral $\begin{gathered}\text { En } \\ \text { desacuerdo }\end{gathered} \begin{gathered}\text { No } \\ \text { respondi } \\ \text { ó }\end{gathered}$

Tengo confianza en mí mismo(a) cuando me enfrento a un problema de matemáticas.

Confío en mí cuando tengo que resolver un problema de matemáticas.

Me considero muy capaz y hábil en matemáticas.

Pienso que podría estudiar matemáticas más difíciles.

Puedo comprender cualquier material impreso que usamos en las clases de matemáticas.

Generalmente tengo dificultades para resolver los ejercicios de matemáticas.

Me angustio cuando el profesor me envía a la pizarra para resolver un problema.

Cuando trabajo con matemáticas mi mente se pone en blanco y soy incapaz de pensar claramente.

Los temas de matemáticas están entre mis

$\begin{array}{rrrr}46,8 & 34,6 & 18,6 & 0,0 \\ 51,0 & 31,8 & 16,6 & 0,6 \\ 41,5 & 29,2 & 28,7 & 0,6 \\ 20,4 & 24,5 & 53,2 & 2,0 \\ 39,1 & 36,2 & 23,3 & 1,4 \\ 37,4 & 32,4 & 29,6 & 0,6 \\ 31,4 & 26,9 & 41,1 & 0,6 \\ 17,0 & 26,9 & 56,1 & 0,0 \\ 19,4 & 21,7 & 58,1 & 0,8\end{array}$


favoritos.

Las matemáticas son muy interesantes para mí.

27,7

28,9

43,3

0,2

Las matemáticas enseñan a pensar.

$68,6 \quad 17,8$

11,7

2,0

Las matemáticas se aprenden rápidamente.

$25,9 \quad 34,8 \quad 37,9 \quad 1,4$

Las matemáticas son fáciles para mí.

$27,1 \quad 31,6$

39,5

Las matemáticas son algo muy abstracto para

mí.

$\begin{array}{llll}21,5 & 41,7 & 35,2 & 1,6\end{array}$

Las matemáticas me hacen sentir nervioso(a).

35,

Los términos y símbolos usados en matemáticas

son difíciles de comprender y manejar.

$\begin{array}{llll}24,3 & 36,2 & 39,5 & 0,0\end{array}$

Me gustaría seguir estudiando más temas de matemáticas,

$\begin{array}{llll}21,5 & 25,1 & 53,0 & 0,4\end{array}$

Para aprender matemáticas no me basta estudiar, sino que me tiene que gustar.

$58,5 \quad 25,1 \quad 16,2 \quad 0,2$

Solo en los exámenes de matemáticas me siento físicamente indispuesto(a),

$30,0 \quad 27,9 \quad 40,7 \quad 1,4$

Fuente: Elaboración propia.

A pesar de lo anterior, la mayoría de los estudiantes señala estar en desacuerdo, al consultarle si pensaba que podría estudiar matemáticas más difíciles, lo que podría significar que confía en sus conocimientos al afrontar situaciones o problemas relacionados con los temas que se desarrollan en la clase, pero no en su capacidad para enfrentarse a otros con un nivel de dificultad mayor. Lo anterior se refuerza al observar que el $56,1 \%$ de los estudiantes indica estar en desacuerdo con el ítem que señala que su mente se queda en blanco cuando trabaja con matemáticas.

Sin embargo, al profundizar en la percepción que tienen los estudiantes de sí mismos en su relación con las matemáticas, la mayoría de los entrevistados señalan tener dificultades con ella e indican que se debe a tres factores principales: la materia en sí, el estudiante y el docente. Al respecto, un estudiante del grupo focal señala

la materia porque a uno desde el principio le puede costar, el profesor porque tal vez no lo explicó de la manera que uno pudiera entenderla mejor y uno porque no puso de su parte tal vez de estudiar en realidad como tenía que haber estudiado esa materia. (Comunicación personal, 25 de octubre de 2012)

Aunque los obstáculos con las matemáticas se atribuyen, en parte, a la dificultad asociada a ella, los estudiantes también mencionan tener responsabilidad. Aspectos como 
poco interés, no hacer las prácticas, no estudiar lo suficiente y falta de compromiso fueron mencionados por los alumnos como obligaciones relacionadas con ellos y que, al no cumplirlas, afectan su rendimiento.

Los estudiantes, incluso, indican que muchas veces en esta clase no prestan la atención requerida y se distraen en otras cosas, lo cual provoca que pierdan las explicaciones y la consideración del docente quien, al ver que no están escuchando, no se interesa por abarcar sus dudas y los "castiga" con su indiferencia.

Cuando los estudiantes alcanzan éxito en la materia, indican, como motivo de esto, factores asociados a ellos, como comprensión de la materia (fácil o les gustaba), esfuerzo, dedicación y suficiente práctica. Ninguno menciona al docente como un factor de su buen desempeño.

A diferencia de lo anterior, el docente sí es mencionado como unos de los factores que afectan, por lo general en forma negativa, el rendimiento en la disciplina. Sus explicaciones constituyen el principal elemento que influye en el proceso de aprendizaje y los alumnos mencionan la falta de compromiso e interés, de algunos, para que ellos comprendan o por emplear distintas formas de presentar los temas. Otros elementos como la asignación de insuficiente práctica, no aclarar dudas y no comprometerse con el estudiante, también fueron mencionados por los alumnos como factores asociados al profesor que les afecta directamente.

En general, los estudiantes, aunque enfatizan en la dificultad de la materia, indican que la problemática con ella no está asociada únicamente a dicha característica, a ellos o al docente, sino que representa una responsabilidad compartida. Por ejemplo, una estudiante, al ser cuestionada con respecto a las causas por las cuales tiene dificultades con la materia, menciona

Di a que no entiendo, porque hay... hay... digamos hay cosas que sí entiendo, hay otras que no. También porque... di porque no sé, el profe a veces explica, pero no sé, yo no le entiendo a veces, en sí yo no le entiendo y a veces también porque uno es vago y no estudia verdad.... o tal vez porque a uno no le da la gana entender, pero di no sé. (Comunicación personal, 01 de noviembre de 2012)

Los estudiantes señalan que ante estas situaciones, se ven obligados a buscar lecciones fuera de la clase y pagar por ellas, esto para lograr obtener un buen resultado en las evaluaciones.

Por una parte, la mayoría de los estudiantes expresa estar de acuerdo con que las matemáticas enseñan a pensar, aunque indica que esta materia no está entre sus temas 
favoritos (58,1\%). Un número importante de estudiantes señala que las matemáticas no les resultan interesantes, no se aprenden rápidamente y no son fáciles. Con respecto a si los hacen sentir nerviosos, el porcentaje de respuesta para las opciones de acuerdo y en desacuerdo difiere muy poco, siendo mayor para este último.

Las matemáticas en sí mismas, como materia de secundaria, representan para los estudiantes una disciplina difícil; dicha característica afecta, directamente, su rendimiento. Ellos reconocen que su estudio, incluso la clase, es distinto a las de otras materias y requiere un trato especial.

Por otra parte, la mayoría de los estudiantes señalan no querer seguir estudiando más temas de matemáticas y que es requisito sentir gusto por esta. Las pruebas escritas fueron detalladas por los estudiantes como un elemento que, en sí mismo, afecta el rendimiento académico al ser un factor que genera presión, nerviosismo, temor y provoca un bloqueo en ellos. Al respecto, un estudiante resume, en su opinión, lo descrito anteriormente:

...la gente pone atención, entiende y el nerviosismo y el temor de salir mal lo hacen salir mal en el examen, porque yo muchas veces he explicado y me hacen todo bien, pero como están en confianza conmigo lo hacen bien, cuando están en el examen, ya están temerosos, y no se acuerdan de nada y el nerviosismo los hace a veces fallar mucho. (Comunicación personal, 30 de octubre de 2012)

Para los estudiantes, el hecho de estudiar mucho no es suficiente para obtener una buena calificación, ya que los exámenes son considerados como una prueba de suerte que no los hace sentir confiados. Al respecto, una alumna en el grupo focal señala:

...uno nunca puede estar seguro. Digamos yo ahora estoy segura de que, según yo, me fue bien en el examen y ahora llego y un 40 seguro, entonces, este di no, o sea eso es lo que a uno lo aburre. (Comunicación personal, 25 de octubre de 2012)

Lo anterior representa un indicio con respecto a la necesidad de implementar otras formas de evaluación que le permitan al estudiante demostrar su aprendizaje. El examen y las pruebas escritas, en general, han sido tipificados como un obstáculo que, aunque no para todo el estudiantado, generan estrés y desmotivación.

Aunado a las pruebas escritas, los estudiantes destacaron que las tareas, como estrategia de evaluación, no son efectivas para ellos, pues los docentes se limitan solamente a revisar si cumplieron o no, pero no muestran interés en el contenido. Desde su perspectiva, esto es un factor que influye directamente en su rendimiento y les genera incertidumbre al no tener una retroalimentación que les indique si lo que realizaron es correcto, qué deficiencias presentan y en qué aspectos deben mejorar. En este sentido, un estudiante señala: “dejan 
tareas y no las revisan en clase entonces uno se lleva respuestas malas para la casa y las estudia mal y eso hace que uno pueda que le va... pueda... hace que le vaya mal en los exámenes". (Comunicación personal, 06 de noviembre de 2012)

Es importante recalcar que el gusto es mencionado por los estudiantes como un factor que afecta, positiva o negativamente, el rendimiento en la materia. Tanto en las entrevistas como en el grupo focal, un importante número de estudiantes indica que no siente agrado por las matemáticas. Un estudiante expresa, con respecto a la disciplina, una opinión bastante común entre los estudiantes. Él apunta que: "Nunca me han gustado. (...) nunca me han llamado ni la atención realmente matemática, siento que es muy... siento demasiado fastidioso matemática, mucho trabajo que va". (Comunicación personal, 25 de octubre de 2012)

La principal causa de lo anterior se debe a las bajas calificaciones que los discentes poseen en la materia, las consecuencias que de esto se han derivado, la falta de aplicaciones de esta en la vida cotidiana (al menos como un recurso didáctico) y la ausencia de una conciencia con respecto a la importancia de su estudio. Por ejemplo, una estudiante indica, que estas:

Sirven pero hay cosas que uno dice para qué las va a utilizar si uno no, no va a estudiar, no va a agarrar tal carrera y no va a ocupar eso verdad. Está bien saber lo básico como sumar y restar y cosas así, pero ¿para qué tanto énfasis en todo eso? En polinomios y esas cosas si es depende de lo que uno va a agarrar verdad, la carrera y a veces uno no lo ocupa. (Comunicación personal, 06 de noviembre de 2012)

Aunado a lo anterior, los estudiantes destacan algunos otros aspectos que influyen en el rendimiento en la materia, como lo es el horario de las lecciones (sugieren que estas deben ser en la mañana) y el tamaño de los grupos, lo que provoca que, al ser secciones con un número elevado de alumnos, que el docente no pueda atender las dudas de todos. Lo anterior lleva como consecuencia que ellos se distraigan, hablen entre sí, se desordene la clase y la comprensión de los temas no sea adecuada. Esta situación fue mencionada por los alumnos en la discusión generada en el grupo focal.

Las matemáticas como un reto fue mencionado por muy pocos estudiantes. Ellos señalan que al ser una materia difícil, según su criterio, resolver un ejercicio y obtener la respuesta correcta es satisfactorio. Una alumna expresa que: "Diay porque si algo es difícil, más bien lo siento como agradable" (Comunicación personal, 30 de octubre de 2012). Es decir, la actitud con que el estudiante afronte el reto influye en el estudio de la materia e 
incluso en su agrado hacia ella, ya sea que lo motive a realizar todas las tareas o le provoque considerarse incapaz de ejecutarlas.

\subsubsection{Componente afectivo}

Al observar los datos de la tabla 3, resulta importante mencionar que la mayoría de los estudiantes considera que las matemáticas son valiosas y necesarias. Sin embargo, aunque se reconoce su utilidad, esta no es evidente en todos los temas de la materia, pues algunos de ellos resultan irrelevantes para los estudiantes. En este sentido, un alumno señala: "Hay cosas de las Matemáticas que a uno tal vez le cuestan entonces uno se pone a pensar "pero esto no me va a servir para nada a mí" (Comunicación personal, 06 de noviembre de 2012).

Tabla No 3. Porcentaje de respuestas dado por los estudiantes encuestados para los ítems asociados con la dimensión aceptación

\begin{tabular}{|c|c|c|c|c|}
\hline Ítem & $\begin{array}{l}\text { De } \\
\text { acuerdo }\end{array}$ & $\begin{array}{c}\text { Neutra } \\
\text { I }\end{array}$ & $\begin{array}{c}\text { En } \\
\text { desacuerd } \\
0\end{array}$ & $\begin{array}{c}\text { No } \\
\text { respondi } \\
\text { ó }\end{array}$ \\
\hline Las matemáticas son valiosas y necesarias. & 67,0 & 21,5 & 10,9 & 0,6 \\
\hline $\begin{array}{l}\text { Me gustan las matemáticas porque cuando hago } \\
\text { mis tareas me satisface encontrar las soluciones. }\end{array}$ & 39,5 & 30,4 & 28,9 & 1,2 \\
\hline Si pudiera no estudiaría más matemáticas. & 46,4 & 19,4 & 33,0 & 1,2 \\
\hline $\begin{array}{l}\text { Las matemáticas que se dan en el colegio sirven } \\
\text { para resolver problemas reales en distintas áreas. }\end{array}$ & 37,2 & 33,0 & 29,1 & 0,8 \\
\hline $\begin{array}{l}\text { Si estudio puedo entender cualquier tema } \\
\text { matemático. }\end{array}$ & 58,1 & 26,1 & 15,4 & 0,4 \\
\hline $\begin{array}{l}\text { Con frecuencia aprendo nuevos conceptos en } \\
\text { matemáticas. }\end{array}$ & 46,6 & 33,2 & 20,0 & 0,2 \\
\hline $\begin{array}{l}\text { Pienso que seré capaz de usar lo que he } \\
\text { aprendido en matemáticas. }\end{array}$ & 44,7 & 35,4 & 19,8 & 0,2 \\
\hline $\begin{array}{l}\text { Disfruto haciendo los problemas que me dejan } \\
\text { como tarea en las clases de matemáticas. }\end{array}$ & 17,6 & 31,0 & 50,8 & 0,6 \\
\hline $\begin{array}{l}\text { Relaciono los nuevos conceptos con las cosas } \\
\text { aprendidas. }\end{array}$ & 32,4 & 37,4 & 29,8 & 0,4 \\
\hline $\begin{array}{l}\text { Solo deberían estudiar matemáticas las personas } \\
\text { que las aplicarán en sus futuras ocupaciones. }\end{array}$ & 46,0 & 19,2 & 34,2 & 0,6 \\
\hline Cualquiera puede aprender matemáticas. & 75,9 & 14,0 & 9,1 & 1,0 \\
\hline Me siento seguro(a) al trabajar con matemáticas. & 31,8 & 39,3 & 27,7 & 1,2 \\
\hline
\end{tabular}


Aunque estudie matemáticas me parecen muy difíciles.

$46,0 \quad 23,9 \quad 28,9 \quad 1,2$

Cuando estudio matemáticas me siento intranquilo(a).

$33,6 \quad 27,1 \quad 38,1 \quad 1,2$

$\begin{array}{lllll}\text { Desearía que las matemáticas no existieran. } & 35,0 & 20,4 & 43,9 & 0,8\end{array}$

Cuando estoy haciendo un examen de matemáticas tengo problemas para resolver problemas o ejercicios que en clase sí podía.

Para mi estudio futuro elegiré una profesión lo más alejada posible de las matemáticas.

Fuente: Elaboración propia.

Un porcentaje considerable de los estudiantes señala que si pudieran no estudiaría esta materia, lo que refleja que la valoración sobre la importancia de esta no implica un interés en continuar su formación en ella. Lo anterior, entre otras causas, debido a lo complicado de la materia y a la valoración que hacen sobre sí mismos con respecto a su incapacidad para estudiar matemáticas más difíciles.

Además, se destaca que la mayoría de los estudiantes indica que si se estudia, se puede entender las matemáticas. Un aspecto que motiva al estudiante en el estudio de la disciplina es la comprensión de la materia y el interés que pueda tener en ella. Si esto no se logra, el estudio de las matemáticas se reduce a un requisito por cumplir, lo que implica estudiar únicamente para pasar los exámenes. En este sentido, una estudiante indica, con respecto a cuál es su motivación para estudiar la disciplina: "que tengo que pasar el año porque es como obligatorio verdad, si uno no estudia no pasa entonces esa es mi motivación, pasar el año, graduarme con mi generación" (Comunicación personal, 01 de noviembre de 2012).

En general, la única motivación que poseen los estudiantes para estudiar matemáticas es aprobar el año y graduarse. Esto se traduce en que sus experiencias positivas en la materia se dan cuando se logra un buen resultado en las pruebas escritas y la promoción en ella.

Para un porcentaje reducido de estudiantes, sin embargo, el posible uso de los conocimientos matemáticos en un futuro, específicamente en carrera universitaria, es su motivación. Por lo tanto, la valoración de la materia y su estudio están en función de las expectativas de formación profesional. 
El porcentaje de respuestas de los estudiantes para la opción de acuerdo en el ítem "Las Matemáticas que se dan en el colegio sirven para resolver problemas reales en distintas áreas" es bajo. Esto constituye un insumo por considerar, pues podría representar un indicio de que los contenidos que se ven en secundaria se desarrollan en forma descontextualizada, sin una aplicación a la vida cotidiana. Esto se vio reflejado al realizar las entrevistas y el grupo focal.

Lo anterior muestra una tendencia en la opinión de los estudiantes quienes, aunque consideran que las matemáticas son útiles, piensan que su estudio, en secundaria, se debería centrar en lo básico, en los contenidos que se emplean diariamente, y que las matemáticas "más complicadas" deberían ser abordadas solo por aquellos que desean realizar carreras universitarias afines a la disciplina. Incluso en el grupo focal los estudiantes señalan que su estudio debería centrarse en saber sumar, restar, multiplicar y dividir.

Es importante señalar que la mayoría de los estudiantes expresa no disfrutar al resolver los ejercicios que se le dejan como tarea en las clases de matemáticas, debido a que no son de fácil comprensión. A la vez, indican que esto se debe a la desmotivación que para ellos provoca este tipo de asignaciones, pues se ve como un requisito académico en sí, pero no como una técnica de aprendizaje o de aplicación de lo aprendido, tal como se señaló anteriormente.

Entre los datos de la tabla 3 se destaca que el $46 \%$ de los estudiantes está de acuerdo en que esta materia solo la deberían estudiar los que la van a aplicar en sus futuras profesiones, lo cual refuerza lo comentado anteriormente. Es importante mencionar que con respecto al ítem Relaciono los nuevos conceptos con las cosas aprendidas, no hay mayoría de respuesta de los estudiantes para ninguna de las opciones. El mayor porcentaje señala tener una posición neutral. Lo anterior podría ser un indicador de que la forma en que se está abordando la materia en secundaria no está permitiendo que los discentes elaboren conexiones con conocimientos previos, incluso con los de otras asignaturas, provocando una imagen parcializada de lo que son y para qué sirven las matemáticas.

Un alto porcentaje de los encuestados indica estar de acuerdo en que cualquiera puede aprender matemáticas. Sin embargo, anteriormente había señalado que el gusto por ellas era un requisito indispensable para su estudio y que solo deberían hacerlo aquellos que las van a utilizar en sus carreras, por lo que podría formular la afirmación que los estudiantes consideran que si se tiene gusto por la materia, se estudia y se aprende, y que es solo para los que la van a utilizar en un futuro. 
Nuevamente el factor emocional asociado a los exámenes surge como un aspecto que genera bloqueo en los estudiantes, pues, aunque señalan confiar en ellos al resolver problemas, la mayoría indica tener dificultades en el momento de la evaluación escrita en ejercicios que habían sido "comprendidos" por ellos. Al respecto, una estudiante señala: "yo por más que intente y estudie, practique, siempre cuando llego al examen, puedo ir bien estudiada (...), y a llegar a la hora del examen es como si no hubiera estudiado nada". (Comunicación personal, 06 de noviembre de 2012)

Los estudiantes destacan que todo el empeño para estudiar la materia, las prácticas realizadas y las aspiraciones para lograr un buen resultado quedan empañadas por el nerviosismo que les afecta al momento la evaluación escrita, lo que les imposibilita realizar de forma correcta los ejercicios propuestos. En los casos más extremos, algunos señalan que dejan las preguntas sin contestar y que, una vez finalizada la prueba, las pueden realizar sin ningún problema.

Una vez analizados los datos obtenidos para el componente afectivo de las actitudes hacia las matemáticas de los estudiantes, a continuación se presenta el análisis de la información adquirida para el componente conductual.

\subsubsection{Componente conductual}

En los datos presentados en la tabla 4, para el componente conductual, se puede observar que los estudiantes expresan que en matemáticas solo se conforman con aprobar, dejando de lado el aprendizaje y la comprensión de los conceptos.

Tabla No 4. Porcentaje de respuestas dado por los estudiantes encuestados para los ítems asociados con la dimensión rechazo

\begin{tabular}{|c|c|c|c|c|}
\hline Ítem & $\begin{array}{c}\text { De } \\
\text { acuerdo }\end{array}$ & Neutra & $\begin{array}{c}\text { En } \\
\text { desacuerdo }\end{array}$ & $\begin{array}{l}\text { No } \\
\text { respondió }\end{array}$ \\
\hline Me gusta participar en clase de matemáticas. & 30,2 & 36,8 & 32,4 & 0,6 \\
\hline $\begin{array}{l}\text { Guardaré mis cuadernos de matemáticas } \\
\text { porque probablemente me sirvan, }\end{array}$ & 45,3 & 25,9 & 28,9 & 0,0 \\
\hline $\begin{array}{l}\text { Me siento motivado(a) en clase de } \\
\text { matemáticas. }\end{array}$ & 24,9 & 40,9 & 33,4 & 0,8 \\
\hline Estudiar matemáticas me aburre. & 45,3 & 25,1 & 29,2 & 0,4 \\
\hline $\begin{array}{l}\text { Me aburro bastante en las clases de } \\
\text { matemáticas. }\end{array}$ & 45,1 & 23,7 & 30,4 & 0,8 \\
\hline Las matemáticas son aburridas. & 41,7 & 24,9 & 32,2 & 1,2 \\
\hline
\end{tabular}




$\begin{array}{lllll}\text { Las matemáticas provocan desánimo. } & 35,6 & 26,7 & 36,0 & 1,8 \\ \text { En matemáticas me conformo con aprobar. } & 52,0 & 15,4 & 29,8 & 2,8\end{array}$

Fuente: Elaboración propia.

Estos datos concuerdan con los comentados anteriormente. Los estudiantes indican que las matemáticas son incomprensibles, por lo que señalan que obtener un bajo rendimiento en ella es normal y lo utilizan como una justificante para los resultados de sus evaluaciones. Incluso mencionan una serie de malestares físicos asociados a ellas como dolor de estómago y náuseas. Algunos indican, con cierto grado de frustración, que no importa el esfuerzo que hagan, siempre van a salir mal e indican la necesidad de ser "muy inteligente" para lograr tener éxito.

\subsection{Actitudes de los docentes hacia las matemáticas}

Debido a que el número de docentes de décimo año en las instituciones participantes fue muy reducido, el análisis de los cuestionarios aplicados se realizó de forma descriptiva, haciendo comparaciones entre las respuestas dadas y complementando estos con los datos obtenidos por medio de la entrevista y observación.

\subsubsection{Componente cognitivo}

Al analizar las respuestas dadas por los docentes para los ítems relacionados con el componente cognitivo, se obtuvo que a estos les gusta interpretar y analizar la información relacionada con las matemáticas que aparece en los medios de comunicación, entender temas complejos con ayuda de la disciplina, tener seguridad de que pueden resolver problemas que requieren un componente matemático, consultar a sus colegas cuando poseen alguna duda y hablar con ellos sobre contenidos de matemáticas. Además, indican, con respecto a la materia, que el reto que representa estar en una clase de esta estimula el desarrollo académico de los estudiantes.

Las docentes entrevistadas son enfáticas en señalar que los estudiantes se muestran desmotivados con respecto al estudio de las matemáticas y que el desarrollo deseado en ellos no se está logrando. Una de ellas, por ejemplo, indica:

Yo creo que ellos mismos se ponen la barrera. La ven como algo muy difícil. Insisto en que la ven como algo importante pero que para ellos no está al alcance y yo creo que ellos la ven no al alcance porque esto también de la tecnología ha afectado un poco. Esta generación cree que con un botoncito todo se soluciona (...). El nivel de 
razonamiento es bastante bajo en ese sentido. (Comunicación personal, 01 de noviembre de 2012)

De acuerdo con los docentes, la actitud del estudiante está relacionada con su rendimiento académico en la disciplina. Una profesora señala al respecto:

Bueno los que son académicamente bien son una actitud bastante positiva. Usted los busca a ellos y ellos inmediatamente responden, usted les pone un problema y ellos ven a ver cómo lo hacen. (...) Hay otros que es una indiferencia (...), una pereza por decirlo así. (Comunicación personal, 06 de noviembre de 2012)

La actitud de los estudiantes es calificada por los docentes como negativa con respecto a las matemáticas. Una de las profesoras entrevistadas indica que algunos por más que se intente y se les llame la atención, no ponen de su parte. Incluso señala que el comportamiento de muchos de ellos en la clase se caracteriza por el desinterés y conformismo, ejemplificado en el uso constante del celular en todo momento, tanto en las explicaciones como en las lecciones de prácticas, así como de ipods y computadoras. Esta situación también fue constatada en la observación efectuada por los investigadores.

La docente señala que el gusto por la materia, la elección de una futura carrera o la influencia de sus mismos compañeros afecta la actitud del estudiante. También manifiesta que la influencia que los alumnos tienen a nivel social, tanto de los medios de comunicación como de los padres de familia, es algo que afecta su actitud. Al respecto, una docente menciona:

...una influencia social siento, usted ve las noticias, usted ve todo y siempre "Matemática la más difícil, hoy es el examen de bachillerato de Matemática, que todo el mundo le tiene miedo y empiezan con tal materia y fue la que salieron más bajo... Uno oye padres de familia en las reuniones es que a mí me costó mate y mi esposo también entonces pobrecita ella y ella le va a costar también". Hay como un perdón social. (Comunicación personal, 01 de noviembre de 2012)

Aunque la actitud de los estudiantes es calificada por los docentes, en general, como negativa, ellos mismos señalan que existen alumnos cuya disposición a la materia resulta muy positiva. Por ejemplo, indican que a algunos les gusta la materia, trabajan bien, estudian por su propia cuenta y se adelantan a sus compañeros, profundizan en algunos temas por medio de la búsqueda de información adicional, participan en olimpiadas de matemáticas, entre otros.

Las docentes entrevistadas indican que se debe trabajar, desde las instituciones, con los estudiantes sobre técnicas de estudio en matemáticas, pues aprender esta materia es 
distinto que con otras disciplinas y los alumnos no son conscientes de ello. Ellos pretenden aprender de forma memorística, pues esto les sirve en otras, pero la diferencia con la disciplina es que si bien la memoria ayuda, se requiere, además, analizar, pensar y entender.

\subsubsection{Componente afectivo}

Para este segundo componente de las actitudes, los docentes indican que las matemáticas son importantes y necesarias y que saber de ellas es trascendental para toda persona, por lo que las consideran fundamentales en la formación de todo ciudadano. En cuanto al uso que ellos les dan a sus conocimientos de la materia, exponen que los utilizan para resolver problemas de la vida cotidiana, permiten ejecutar distintas actividades adicionales a su trabajo, les proporcionan información para tomar decisiones, relacionan sus conocimientos con los de otras áreas y que se sienten más cómodos al trabajar con algunos temas que con otros. Sin embargo, cuando se les consultó si las matemáticas facilitan la comunicación de la información diaria, una docente indicó tener una opinión neutral, aunque los otros tres docentes señalaron estar de acuerdo. Si bien los profesores apuntaron que la materia es importante en la formación de toda persona, resulta interesante observar que, al cuestionarles si deberían estudiarla solo aquellos que las aplicarán en sus futuras carreras, un docente señala tener una posición neutral.

Con respecto a la pregunta relacionada con que si esta disciplina es difícil para muchos estudiantes, tres docentes señalaron estar de acuerdo. Cuando se les consultó sobre la aplicabilidad de los contenidos, solo un profesor señaló estar de acuerdo en que no todo se puede utilizar en la vida cotidiana, aunque anteriormente había indicado utilizarlas para resolver problemas.

En la entrevista, las docentes consultadas reconocen, tal como se evidenció en los datos obtenidos en el cuestionario, la importancia de las matemáticas. Sin embargo, aceptan que en el aula tal aplicabilidad no se presenta, aunque está en las propuestas del MEP. Esta aplicabilidad de la disciplina tampoco es evidente en los estudiantes: pues una docente señala:

En la mayoría de los casos una pregunta muy común es para qué me sirve eso, yo voy a estudiar tal cosa, para qué me sirve, qué pereza, eso no sirve para nada, con que uno sume, resta, divida es ya suficiente para ellos, según ellos. (Comunicación personal, 01 de noviembre de 2012)

Esto indica la ausencia de estrategias para mostrarles a los estudiantes la aplicabilidad de las matemáticas no solamente en asuntos de la vida cotidiana, la ciencia y la tecnología, 
sino como una disciplina que contribuye al desarrollo de estrategias cognitivas, de razonamiento, uso de lenguaje y argumentación Una docente entrevistada enfatiza que, algunas veces, son los mismos profesores quienes no han asumido el reto de enseñarles a los estudiantes la aplicabilidad de la materia y que más bien se ha reducido a una enseñanza algorítmica y memorística. En este sentido, añade que el docente debe buscar estrategias para motivar a sus alumnos y que estos valoren la importancia de la disciplina, pues muchas veces las aplicaciones no son evidentes y requieren de mucha planificación por parte del profesor.

\subsubsection{Componente conductual}

De los ítems redactados para este último componente de las actitudes, se obtuvo que los docentes indican sentir agrado por trabajar con matemáticas, desean aprender más sobre la disciplina y que, con frecuencia, tratan de hacerlo, por lo que les gustaría profundizar en temas relacionados con ella.

Con respecto a sí mismos ante la disciplina, señalan sentirse seguros para trabajar con cualquier tema de matemáticas en su ejercicio profesional, cuando alguien les solicita analizar una situación real aplicando sus conocimientos y que pueden resolver ejercicios de la materia cuya complejidad es alta para la mayoría de las personas; además de lo anterior, apuntan sentirse motivados cuando asisten a alguna actividad relacionada con las matemáticas.

No todos los docentes estuvieron de acuerdo en que las matemáticas sirven para resolver problemas en distintas áreas, respuesta que está relacionada con su percepción de que muchos de los contenidos de esta materia no tienen una aplicación en la vida cotidiana. De igual forma, no hubo consenso de todos al consultarles si cualquiera puede aprender matemáticas. Sin embargo, en la entrevista sí indicaron que esto es posible, siempre y cuando se dedique y estudie lo suficiente, aunque por las cualidades de algunos estudiantes se les dificulta más que a otros y requieren un poco más de esfuerzo. De acuerdo con la opinión de los docentes, esta materia constituye un factor para la escogencia de carrera.

En relación con los requisitos que debe cumplir un estudiante para ser bueno en matemáticas, señalan que el gusto por la materia, la dedicación, disciplina y los buenos hábitos de estudio son fundamentales para ello. Una mente positiva también influye. Una docente, además, comenta que la concentración en la clase es primordial para comprender. En su opinión, si un alumno escucha con atención la lección, esto favorece su rendimiento académico; sin embargo, la realidad en las aulas es distinta y estos se distraen con mucha 
facilidad, ya sea por la desmotivación o por la presencia de diversos elementos que captan su atención.

Además de lo anterior, una docente destaca que el estudiante debe "creérselo", confiar que es capaz de estudiar Matemáticas y aprobar, porque, según ella, "hay que verlos el día que hacer examen viene "Es que me voy a quedar, es que no puedo, es que se me olvida". Yay no han empezado el examen y ya vienen con una mentalidad de que lo perdí" (Comunicación personal, 30 de octubre de 2012). Por esta razón, destaca la importancia de una actitud positiva del estudiante que le favorezca disponerse para llegar a clase, poner atención, tener confianza en sí mismo y no menospreciar sus capacidades.

\section{Creencias hacia las matemáticas}

Para el caso de las creencias hacia las matemáticas, los datos se presentan según la categorización de estas indicada en el apartado 2.

\subsection{Creencias de los estudiantes hacia las matemáticas}

\subsubsection{Creencias sobre las matemáticas}

Los datos se presentan por cada una de las visiones de la disciplina. De acuerdo con las características asociadas a la visión instrumentalista de las matemáticas, la mayoría de los estudiantes está de acuerdo en que esta disciplina provee conocimiento a otras áreas y que se trata de manipular números y símbolos.

Para la segunda visión, la platónica, solamente para el ítem relacionado con la unicidad de las soluciones para los problemas de matemáticas, se presentó mayoría de respuestas de los estudiantes para la opción de acuerdo. Es importante indicar que, en esta visión, un porcentaje importante de alumnos considera que en la disciplina lo que importa es el resultado final y no necesariamente el proceso realizado para ello.

En los ítems asociados con la visión de resolución de problemas de las matemáticas, los estudiantes concuerdan, en mayoría de respuestas, que en los problemas de esta disciplina hay distintas formas de encontrar una solución, que en estas aún quedan muchas cosas por descubrir, que son útiles para resolver dificultades de la vida cotidiana y valoran el error como una parte importante en su aprendizaje.

Con el propósito de conocer la visión de las matemáticas que poseían los participantes, se sumaron los puntajes dados por ellos a las características de cada una de las visiones. Aquella en la cual el alumno obtuvo el mayor puntaje fue con la que se le asoció. Es 
importante señalar que algunos de los encuestados presentaron este valor en dos visiones, aunque el porcentaje de estudiantes en esta condición fue muy pequeño.

Según los datos de la tabla 5, ninguna de las visiones obtuvo una mayoría por parte de los estudiantes encuestados. Sin embargo, es la visión de resolución de problemas en donde se ubica el mayor porcentaje de alumnos.

Tabla No 5. Porcentaje de estudiantes que se ubican en las distintas visiones de las matemáticas

\begin{tabular}{lc}
\hline \multicolumn{1}{c}{ Visión } & $\begin{array}{c}\text { Porcentaj } \\
\text { e }\end{array}$ \\
\hline Resolución de problemas & 38,5 \\
Instrumentalista & 31,4 \\
Platónica & 21,5 \\
Instrumentalista-resolución de problemas & 7,9 \\
Platónica-resolución de problemas & 0,4 \\
Instrumentalista-platónica & 0,2 \\
Total & 100,0 \\
\hline
\end{tabular}

Fuente: Elaboración propia.

Esto implica que un número importante de estudiantes considera que hacer matemáticas es investigar nuevas ideas y concibe a esta disciplina en continua expansión, cambiante, que puede ser objeto de revisión y que es útil para resolver dificultades cotidianas. Además, destaca que para resolver los problemas en la disciplina no hay un único método y se valora el error como una forma de aprendizaje en la materia.

Según la visión de las matemáticas que poseen los estudiantes, en general, se destacan las características de difíciles, complicadas, aburridas, mecánicas, rígidas, exactas, que no se pueden cambiar, aunque están en continua expansión, asociadas con exámenes de convocatoria y que generan temor. Una alumna, por ejemplo, señala: “...la verdad es que ya cuando a uno le dicen Matemática uno es como 'me va a ir mal'”. (Comunicación personal, 30 de octubre de 2012)

Aunado a lo anterior, resulta interesante el hecho de que los estudiantes, en el grupo focal, indican que esta materia es un "coladero" y que representa un filtro para que no todos puedan estudiar. Desde este punto de vista, no se visualiza la materia como un medio para el desarrollo de habilidades lógicas y de razonamiento, sino un "obstáculo en su camino". 
Además, destacan que es una materia distinta a las otras y ello se refleja también en su estudio. Entre las características que ellos mencionan para diferenciarla de otras es que para comprenderla es necesario disponer de atención en el aula, en contraste con otras en las que, aunque no se perciba en la lección habitual, se puede llegar a entender con leer 0 repasar los apuntes tomados.

Los alumnos que señalan sentirse identificados con una imagen positiva de las matemáticas se enfocan en señalar que esta materia no es tan difícil, no les despierta temor; se sienten bien cuando obtienen un buen resultado en una evaluación; expresan sentir satisfacción al resolver problemas de la materia, aunque sean difíciles; han tenido buenos profesores, practican constantemente y mantienen una buena disposición.

Como es posible observar, aunque la mayoría de los estudiantes asocia a las matemáticas con características negativas, existen otros para los cuales estas son agradables. Por lo tanto, no se puede generalizar que para todos los alumnos la disciplina representa un obstáculo, una dificultad o les genera rechazo.

\subsubsection{Creencias de los individuos con respecto a sí mismos en matemáticas}

A pesar de la dificultad que los estudiantes asocian con las matemáticas, esta característica no es la única determinante para su bajo rendimiento en la materia. Indican que existen factores, asociados a sí mismos, que afectan su desempeño en ella. Por ejemplo, un alumno indica: "A mí la Matemática no me ha hecho nada. Si he salido mal o si algo ha sido es por vagabundería o cosas mías" (Comunicación personal, 01 de noviembre de 2012). Los estudiantes mencionan que el poco esfuerzo o interés, no hacer ejercicios, no estudiar o practicar lo suficiente, falta de concentración y cuidado al momento de realizar el examen, miedo, inseguridad, temor, tanto a la materia como a su evaluación, y malas bases de años anteriores son factores que afectan su rendimiento. Para algunos alumnos esto provoca un bloqueo.

En la tabla 6 se presenta las respuestas para los ítems asociados a la imagen de sí mismos en matemáticas.

Tabla No 6. Porcentaje de respuestas dado por los estudiantes encuestados para los ítems asociados con las creencias de los individuos respecto a sí mismos en matemáticas

$\begin{array}{lcccc}\text { Ítem } & \text { De } & \text { En } & \text { No } \\ \text { acuerdo } & \text { Neutral } & \text { desacuerd } & \text { respondi } \\ & & 0 & \text { ó }\end{array}$


Creo que este año recibiré una excelente nota en matemáticas.

Puedo comprender incluso las cosas más difíciles que nos dan en clase de matemáticas.

Yo soy bueno(a) en matemáticas.

Si trabajo duro entonces puedo comprender toda la materia de matemáticas.

Tengo confianza en mi capacidad para resolver cualquier problema de matemáticas.

Estoy seguro(a) de que aprobaré matemáticas, independientemente del o la profesora que tenga.

Las matemáticas son un tema difícil.

Para aprobar matemáticas hay que ser muy inteligente,

$\begin{array}{llll}28,7 & 23,9 & 46,2 & 1,2 \\ 22,3 & 31,2 & 46,2 & 0,2 \\ 30,8 & 28,9 & 38,3 & 2,0 \\ 74,7 & 16,6 & 8,1 & 0,6 \\ 53,8 & 28,3 & 16,8 & 1,2 \\ 50,6 & 21,3 & 27,3 & 0,8 \\ 50,4 & 25,9 & 21,3 & 2,4 \\ 20,0 & 31,4 & 47,0 & 1,6\end{array}$

Fuente: Elaboración propia.

Según los datos anteriores, los estudiantes consideran que el comprender la materia está asociado al grado de esfuerzo y trabajo que se haga en ella. Por lo tanto, si se "trabaja duro" se puede comprender los contenidos. Aunado a lo anterior, apuntan tener confianza en sí mismos al resolver cualquier problema de matemáticas y que su aprobación en la materia es independiente del profesor. Aunque el porcentaje de respuesta no es mayoría, pero sí el mayor de las opciones disponibles para los ítems, señalan estar en desacuerdo con las afirmaciones Creo que este año recibiré una excelente nota en matemáticas, Puedo comprender incluso las cosas más difíciles que nos dan en clase de matemáticas y Yo soy bueno(a) en matemáticas. Es decir, que la confianza que expresan en algunos ítems no se ha visto reflejada en la visión de sí mismos, su rendimiento y entendimiento de la materia.

Aunque un porcentaje considerable de estudiantes considera que el ser inteligente no es un requisito indispensable para aprobar en matemáticas, pues señala que esto depende más del "trabajo" que se haga en la disciplina, en la entrevista sí lo señala como un aspecto importante, pero no determinante.

Aunado a los datos anteriores, los alumnos profundizaron, según su opinión, con respecto a los requisitos que se deben cumplir para ser un buen estudiante de matemáticas. Ellos mencionan que se debe prestar mucha atención, realizar un gran esfuerzo, controlar los nervios, tener interés por el estudio de la materia, pensar, aclarar las dudas con el profesor, verificar que las respuestas sean correctas y practicar mucho; este último aspecto 
fue señalado por la mayoría de los estudiantes en la entrevista. Ellos mencionaron los aspectos anteriores cuando se les cuestionó acerca de los factores o elementos que asociaban con un buen rendimiento.

Cuando los resultados no fueron satisfactorios para el estudiante, a pesar de todo su esfuerzo, los sentimientos expresados por ellos fueron negativos. Tristeza, frustración, presión, pensamientos nocivos y desánimo fueron emociones mencionadas por los alumnos cuando el resultado en una evaluación fue bajo y la "culpa" de esto no estaba relacionada con ellos. Incluso un estudiante, en el grupo focal, señala que cuando empezó a tener bajos resultados en la materia ir a esa clase era un "infierno" y comenzó a tener pensamientos como "qué pereza, todo me va a salir mal y nada me va a dar" (el resolver un ejercicio).

Asociado a lo anterior, un alumno señala que cuando obtiene una mala nota, se siente mal, pero realiza la diferencia en su sentir cuando considera que es por su culpa y cuando no. Él señala

...si estudié mucho para eso y me dio mala nota, ahí es cuando digamos... eh eh eh... me... me deprimo un poco más porque sé que tengo que estudiar ahora el doooble para ver si mejoro la nota porque no lo entendí a pesar de que yo pensé que lo había entendido o así. Y cuando es que... digamos yo no... yo no estudié o que o que fue por vago o estaba disperso, ahí tan... no me preocupo tanto tanto porque sé que digamos... que si voy estudiando o si me pongo a repasarla ya después lo puedo entender. (Comunicación personal, 30 de octubre de 2012)

El grado de comprensión de los contenidos es otro de los factores que influyen en la disposición del estudiante. Por ejemplo, dos estudiantes indican que cuando no comprenden los contenidos en la clase, se frustran, les da pereza estudiar y se sienten presionadas porque ven "en peligro" su aprobación. Incluso una de ellas indica que no comprenderla y no poder realizar los ejercicios asignados influyó en su gusto por las matemáticas. Entenderla e incluso salir bien con respecto a sus compañeros es un motivo para que el estudiante se sienta bien y capaz en la materia. El gusto por ella está estrechamente relacionado con el grado de entendimiento. Sin embargo, no para todos las matemáticas son "malas". Algunos, los que han obtenido buenos resultados, generalmente, indican que la materia no les genera ningún sentimiento negativo, por lo que su percepción de ella es muy favorable.

\subsubsection{Creencias sobre el papel del profesor dentro del contexto de la clase}


De acuerdo con el papel del profesor dentro del contexto de la clase, se les preguntó a los estudiantes sobre la tendencia didáctica de este, la imagen de lo que consideraban era un buen profesor y la percepción del docente.

Con respecto a la tendencia didáctica, es importante señalar que las preguntas no fueron directas. Es decir, se les presentaba a los alumnos una serie de características o comportamientos de los docentes y se les solicitaba indicar con cuáles identificaban a su profesor. Con base en sus respuestas se determinó en cuál tendencia didáctica se ubicaba el docente.

De los ítems asociados con la concepción tradicionalista, los estudiantes señalan, en mayoría, que los docentes de matemáticas enseñan reglas y procedimientos por seguir y que la metodología que estos siguen es ilustrar, paso a paso, cómo resolver algunos problemas para, posteriormente, plantear ejercicios similares.

Aunque para el ítem El o la profesora de matemáticas evalúa el aprendizaje únicamente con exámenes no hubo mayoría de respuesta en el cuestionario en ninguna de las opciones, tanto en las entrevistas como en el grupo focal los estudiantes señalaron que la evaluación en matemáticas se basa en pruebas escritas. De acuerdo con la opinión de ellos, este es el instrumento más utilizado y no les han aplicado otras formas de valoración.

También señalan que les asignan tareas y, en algunos casos, pruebas cortas. Una queja generalizada es que la evaluación que el docente realiza no tiene correspondencia con los ejemplos vistos en clase. Indican, según su opinión, que el grado de dificultad de los ejercicios que el profesor incluye en una prueba escrita es mayor a los que se realizan en clases o se incluyen en las prácticas, por lo que no tienen las destrezas suficientes para enfrentarse a ellos.

Con respecto a las características de la tendencia didáctica tecnológica, únicamente para una de ellas los estudiantes señalaron, en mayoría, estar de acuerdo. Los estudiantes expresan que los profesores de matemáticas se interesan por conocer los procesos por medio de los cuales se llega a una respuesta, lo que presenta un indicio que la importancia para ellos no recae en la respuesta en sí..

En relación con las características de la tendencia didáctica espontaneísta, solamente dos de ellas recibieron, en mayoría, una respuesta positiva por parte de los estudiantes. Los encuestados apuntan que sus profesores de matemáticas escuchan las inquietudes, comentarios o dudas que ellos plantean y que enfatizan en la necesidad de comprender los contenidos que se desarrollan en la clase y no su memorización. Esto representa una contradicción con lo expresado anteriormente, pues indicaron que la enseñanza empleada 
por ellos se basa en un enfoque algorítmico que incentiva la memoria. Un aspecto que recibió mayoría de respuestas para la opción en desacuerdo fue el uso de la técnica de trabajo en grupo, la cual no es muy utilizada por los docentes.

Con respecto a la tendencia didáctica investigativa, ninguno de los ítems obtuvo mayoría de respuesta por parte de los estudiantes, por lo que se podría decir que las prácticas que describen a esta no son aplicadas frecuentemente por los docentes.

Finalmente, con el fin de clasificar la tendencia didáctica de los profesores de matemáticas, según la opinión de los estudiantes, se presentan los datos de la tabla 7.

Tabla No 7. Porcentaje de estudiantes que clasifican la tendencia didáctica de su profesor como tradicionalista, tecnológica, espontaneísta o investigativa

\begin{tabular}{lc}
\hline \multicolumn{1}{c}{ Tendencia didáctica } & General \\
\hline Tradicionalista & 41,1 \\
Tecnológica & 21,7 \\
Espontaneísta & 14,4 \\
Investigativa & 14,8 \\
Otras & 8,0 \\
Total & 100,0 \\
\hline
\end{tabular}

Fuente: Elaboración propia.

Como se puede observar en la tabla anterior, ninguna de las tendencias didácticas recibió mayoría de respuestas de los estudiantes. Sin embargo, es la tradicionalista la que posee el mayor porcentaje de respuestas por parte de los alumnos, lo cual se vio reflejado tanto en las entrevistas como en la observación de las lecciones.

La metodología empleada por los docentes es descrita por los estudiantes como rutinaria, basada en ejemplos, prácticas y aclaración de dudas cuando se puede. Una estudiante la describe de la siguiente forma

Bueno cuando se empieza un tema... eh eh eh... primero se introduce, se explica más o menos de qué se trata, luego van a la pizarra, lo desarrollan... el primer ejemplo, luego el segundo ejemplo, luego varios ejemplos para que el estudiante pueda entender... eh eh eh ... luego preguntan que si hay dudas, luego ponen prácticas y... las desarrollamos, vemos las malas y las buenas. (Comunicación personal, 06 de noviembre de 2012)

Con respecto a la forma en que el profesor aborda la clase es interesante que, aunque los alumnos indican que las matemáticas es una materia mecánica, y critican este hecho, 
abogan por una enseñanza algorítmica que les permita, por medio de una serie de pasos, llegar a la respuesta correcta dejando de lado procesos de razonamiento y argumentación lógica.

Según sus respuestas, la práctica educativa del docente se puede caracterizar como sigue:

1) De acuerdo con la metodología empleada en la clase, los docentes primero muestran, paso a paso, cómo se deben resolver los problemas o ejercicios específicos, para luego dar una lista de otros similares.

2) Su enseñanza se caracteriza por la presentación de reglas y procedimientos para que los estudiantes los aprendan, sigan y puedan aplicarse en ejercicios posteriores.

3) Muestran interés, no solo por la respuesta final obtenida por los estudiantes en un ejercicio, sino, además, en el procedimiento empleado por ellos para esto.

4) Manifiestan interés por las consultas y comentarios que hacen los estudiantes durante el desarrollo de la clase.

5) Buscan la comprensión de los contenidos matemáticos y no la memorización de estos.

6) No incentivan el trabajo en grupo, sino la escucha atenta por parte de los estudiantes de lo explicado en la clase y, luego, el trabajo individual en la resolución de ejercicios similares a los planteados por los mismos docentes.

A los estudiantes, a la vez, dentro de este contexto, se les consultó en relación con las características que ellos consideraban debía tener un profesor de matemáticas. Para los estudiantes encuestados saber explicar los contenidos matemáticos es un requerimiento esencial, lo cual va aunado a que este debe "saber" sobre la materia que enseña. Además , escuchar y atender la parte emocional y cognitiva de los estudiantes (motivación, interés, esfuerzo, creación de herramientas para el aprendizaje) es una característica deseada; aunque indican que no necesariamente deben resolver todos los ejercicios, sí señalan la importancia de que se ejemplifique con prototipos que permitan tener una visión de todos los casos posibles y que aporten herramientas para enfrentarse a algunos similares.

Entre los requisitos fundamentales para la valoración de un docente de matemáticas como uno bueno están que sus explicaciones sean claras y que asigne bastantes prácticas. Aspectos como la aclaración de dudas, que se preocupe por los alumnos y la realización de centros de estudio fueron mencionados sin que hubiera una opinión generalizada como en los dos aspectos anteriores.

Según la percepción del docente de matemáticas por parte de los estudiantes, la mayoría señala que este comete errores y los enmienda, se interesa por ayudarles con sus 
dificultades en la materia, muestra dominio de los contenidos que explica, está atento a las dudas y comentarios de ellos, usa un lenguaje claro al comunicarse y lo consideran diferente al de otras asignaturas.

Algunos alumnos comparan el trato de los docentes del colegio con respecto a la escuela y destacan la diferencia con un ambiente más maternal, donde se pretendía que ellos se interesaran por las matemáticas, y uno donde no se les presta tanta atención, lo que les hace sentirse solos. Una estudiante al respecto señala:

No sé... como en la escuela... en la escuela explicaban más... este... más prácticas... le ayudaban a uno si no entendía, digamos, lo llamaban a uno "usted entiende o no entiende", "que tal cosa, venga y yo le ayudo" cosas así. Aquí no. Obviamente aquí es diferente verdad, en el cole es diferente y aquí como que cada uno di verdad agarre para su saco. (Comunicación personal, 06 de noviembre de 2012)

En relación con la influencia que los docentes de matemáticas han tenido para que los estudiantes se sientan identificados con las imágenes negativas respecto a la materia, estos señalan que es porque los profesores "son malos", debido a que no explican lo suficiente para comprender la materia, por lo que se ven obligados a buscar clases particulares; además, apuntan a que no se interesan porque se logre una adecuada comprensión, no los motivan, sienten que cuando un alumno ha tenido malas calificaciones, el docente pierde el interés en apoyarlos y que la metodología utilizada no contribuye con su aprendizaje.

Es importante mencionar que no todos indican que el docente haya influido, positiva o negativamente. Algunos mencionan que les va mal porque "las matemáticas son difíciles" por lo que "el efecto profesor" es nulo o muy poco.

Con base en las situaciones indicadas por los alumnos, ellos, a la vez, comentan una serie de requerimientos para los docentes de la disciplina. Debido a que ven a las matemáticas como mecánicas o algorítmicas, ellos claman por una enseñanza que les permita tener un método infalible para todos los casos; a la vez, consideran que la instrucción de la disciplina debe ser un proceso que vaya de lo más simple a lo más complejo, lo que les permitiría comprender, en forma progresiva, los contenidos matemáticos.

Además, enfatizan en que los docentes deben explicar más, estar pendiente de los estudiantes; preguntarles si tienen dudas y aclararlas, ordenar los ejemplos en la clase según grado de dificultad; motivarlos, dar las respuestas de las prácticas, revisar el procedimiento y los resultados de los ejercicios dados como tareas; interactuar más con ellos, darles un trato personalizado, hablarles; conocerlos, establecer confianza con los 
alumnos, realizar centros de estudio, buscar una forma neutra de explicar que permita que todos comprendan la materia; crear un ambiente más cómodo en la clase, explicar ejemplos en los cuales se muestren todos los casos posibles de manera sencilla; asignar suficientes prácticas e implementar actividades lúdicas como una estrategia de enseñanza.

De igual forma, exponen la necesidad de que el profesor no evalúe únicamente basado en el examen, sino que aplique una prueba "cualitativa" en la cual se reconozca el esfuerzo e interés mostrado por el estudiante y se adopten otras formas de verificar los aprendizajes.

En la siguiente sección, se presentan los resultados obtenidos con los profesores para esta segunda variable; luego se contrastan los hallazgos de ambos grupos participantes.

\subsection{Creencias de los docentes hacia las matemáticas}

\subsubsection{Creencias sobre las matemáticas}

De las características de las matemáticas desde una visión instrumentalista, los docentes señalan estar de acuerdo con que estas proveen conocimientos que se utilizan en otras ciencias, que son importantes porque son útiles en la vida, son usadas para modelar situaciones reales, capacitan para comprender mejor el mundo y que las personas las utilizan en su vida cotidiana. Además, coinciden en estar en desacuerdo con la afirmación de que todo en matemáticas es aplicar fórmulas.

Según la visión platónica de las matemáticas, los docentes señalaron estar en desacuerdo con que aprender la disciplina significa principalmente memorizar, que todos los temas de ella están creados y nada más pueden ser construidos, que hay una sola forma de pensar la solución correcta de un problema, que la materia son conceptos y procedimientos que se deben memorizar, que lo único importante es el resultado final y es una pérdida de tiempo cuando se les hace pensar solos sobre cómo se resolvería un nuevo problema.

Dadas las características de la disciplina desde una visión de resolución de problemas, los docentes coincidieron con que las matemáticas son una forma de pensar usando símbolos y ecuaciones, que en los problemas hay diversas formas para llegar a encontrar una solución correcta, que están en continua expansión y muchas cosas quedan aún por descubrir, son útiles para interrogantes cotidianas y que cometer errores es una parte importante del aprendizaje de la disciplina.

En la tabla 8 se presenta el promedio (de 1 a 5 ) obtenido por cada uno de los docentes para las distintas visiones de las matemáticas. De acuerdo con los datos es posible observar 
que la visión predominante es la de resolución de problemas, pero señalan importantes características de la visión instrumentalista.

Tabla No 8. Promedio obtenido por los docentes en cada una de las visiones de las matemáticas

\begin{tabular}{lcccc}
\multicolumn{1}{c}{ Visión } & Docente 1 & Docente 2 & $\begin{array}{c}\text { Docente } \\
\mathbf{3}\end{array}$ & $\begin{array}{c}\text { Docente } \\
\mathbf{4}\end{array}$ \\
\hline Instrumentalista & 4,25 & 3,63 & 3,50 & 3,75 \\
Platónica & 1,71 & 1,29 & 1,86 & 1,00 \\
Resolución de problemas & 4,38 & 4,13 & 3,50 & 4,88 \\
\hline
\end{tabular}

Fuente: Elaboración propia.

En general, señalan que matemáticas es investigar nuevas ideas, que hay diversas formas para encontrar la solución correcta, que son usadas para modelar situaciones reales, están en continua expansión, son útiles en la vida para resolver problemas cotidianos, son una forma de pensar usando símbolos y ecuaciones, proveen conocimientos que se emplean en otras ciencias, capacitan para comprender mejor el mundo, pues el error es una parte importante del aprendizaje de la disciplina, y que pueden ser objeto de revisión para identificar inconsistencias.

\subsubsection{Creencias de los individuos con respecto a sí mismos en matemáticas}

Con respeto a sí mismos y su relación con la disciplina, los docentes señalan estar de acuerdo con que el trabajo en grupo les facilitaba el aprendizaje de las matemáticas y se consideran buenos en la materia.

Donde no hubo coincidencia en el grado de acuerdo fue para el ítem del cuestionamiento relacionado con la valoración de obtener una excelente nota si se matricularan en un curso adicional de la disciplina. Fue posible observar que, aunque los docentes indican sentir confianza con respecto a la materia, no confían en su capacidad para continuar con el estudio de esta o de contenidos ajenos a su labor diaria.

\subsubsection{Creencias sobre el papel del profesor dentro del contexto de la clase}

De acuerdo con la didáctica que caracteriza la labor del docente, a los profesores se les cuestionó con cuáles de las características de la tendencia tradicionalista, tecnológica, espontaneísta e investigativa se identificaban más. 
Primero, para cada una de las características debían señalar con cuál se edificaban en una escala de 1 a 5 . Posteriormente, se obtuvo un promedio del valor dado a los ítems, según la tendencia didáctica asociada, para analizar en cuál se presentaba el mayor puntaje. En la tabla 9 es posible observar la tendencia en la cual cada docente presentó un mayor puntaje.

Tabla No 9. Promedio obtenido (de 1 a 5) por los docentes en cada una de las tendencias didácticas

\begin{tabular}{lcccc}
\multicolumn{1}{c}{ Visión } & $\begin{array}{c}\text { Docente } \\
\mathbf{1}\end{array}$ & $\begin{array}{c}\text { Docente } \\
\mathbf{2}\end{array}$ & Docente 3 & $\begin{array}{c}\text { Docente } \\
\mathbf{4}\end{array}$ \\
\hline Tradicionalista & 3,60 & 3,40 & 2,40 & 2,80 \\
Tecnológica & 3,40 & 4,20 & 3,80 & 4,80 \\
Espontaneísta & 4,29 & 4,00 & 4,57 & 4,43 \\
Investigativa & 3,75 & 4,25 & 3,50 & 3,50 \\
\hline
\end{tabular}

Fuente: Elaboración propia.

Es posible detallar en la tabla que, según la valoración realizada por los docentes, estos no se identificaron con las características de la tendencia didáctica tradicionalista. Según las características de la tendencia tecnológica, todo el profesorado está de acuerdo con que le interesan los procesos por medio de los cuales los estudiantes llegan a una respuesta y piensa que los errores están bien y son buenos para el aprendizaje.

Acerca de la tendencia espontaneísta, indica su deseo de que los alumnos estén a gusto cuando aprenden nuevos conocimientos en matemáticas, escucha atentamente cuando los estudiantes preguntan o dicen algo durante la clase y quiere que ellos comprendan los contenidos matemáticos que se desarrollan en las lecciones, no que los memoricen. Además, señala que comprende los problemas y las dificultades que ellos experimentan en la disciplina y que realiza bastantes trabajos en grupo. Relacionado con este último punto, hay una contradicción con respecto a lo que sucede en el aula, pues tanto en la observación como en las entrevistas y grupo focal de los estudiantes se constató que esta técnica es muy poco empleada en las clases.

Por último, de la tendencia didáctica investigativa los profesores apuntan que estimulan distintos procesos de solución, por parte de los estudiantes, a las actividades propuestas en el aula e indican sentirse contentos cuando sus alumnos se esfuerzan, aunque los resultados no sean los esperados.

De acuerdo con la opinión del cuerpo docente, hacer énfasis en el uso de la memoria para aprender los contenidos y procedimientos, evaluar el aprendizaje de los estudiantes 
únicamente con exámenes, basarse en el libro de texto para impartir las clases, dar tiempo a los alumnos para explorar los nuevos problemas matemáticos y plantear actividades de investigación para que ellos adquieran los contenidos matemáticos son aspectos que no caracterizan su labor en el aula. Es interesante observar que, aunque los docentes indican no basarse en una metodología tradicional, muchas de las actividades que se realizan en el aula sí poseen estas características, lo cual se vio reflejado tanto en la observación como en las entrevistas.

Una docente, por ejemplo, al consultarle qué actividades desarrollaba en el aula para enseñar los contenidos de matemáticas, indicó que se basaba en ejemplos, problemas, prácticas, la pizarra, el libro y la calculadora. Indica que, aunque ha intentado realizar juegos en la clase, ha sido prácticamente imposible una eficaz aplicación de ellos, pues los estudiantes no responden, les da pereza y no prestan atención, por lo que ha desistido de ello. También señala las dificultades que se presentan para emplear la tecnología y utilizar un laboratorio de cómputo, pues lo prestan únicamente para dos lecciones y no hay posibilidad de utilizarlo para todos los grupos como una herramienta en el aprendizaje de la disciplina. Sin embargo, asegura la necesidad de utilizar distintas metodologías para llamar la atención del estudiante.

Una docente afirma y defiende que la clase magistral es una forma efectiva de enseñar y que ella aprendió así, por lo que la utiliza. Ella señala:

Bueno soy muy tradicional. Yo elaboro la teoría. Trato de que sea la menos posible y a partir de ahí doy algunos ejemplos que le permitan al estudiante descubrir qué es el objetivo que se quiere alcanzar con eso. Los ejercicios para mí siguen siendo fundamentales. (Comunicación personal, 15 de noviembre de 2012)

Con respecto a las formas de evaluación, los docentes manifiestan que aplican las que señala el MEP, como tareas, trabajos extraclase y exámenes. Sobre las tareas una docente opina, con un poco de frustración, lo difícil que los estudiantes se tomen estas con responsabilidad, pues ellos no realizan dichas asignaciones, sino que las copian. Una docente apunta que: "Por lo general habían (sic) copias. Uno lo notaba. E incluso... bueno el primero lo tuve que evaluar así por... presentación casi. Ya para el segundo agregué una nota, una observación, a partir del segundo que puse así que iba a realizar comprobaciones orales y a partir de ahí les bajaba un 1\% y más de uno lo terminaba perdiendo ese $1 \%$ verdad". (Comunicación personal, 13 de noviembre de 2012)

Los docentes apuntan un aspecto que, para ellos, es muy preocupante. Para ellos, los alumnos tienen memoria a corto plazo y estudian únicamente para un examen, después de 
eso todo lo olvidan. Una docente, al respecto, dice: “...usted les pone una factorización y no saben, les ponen una ecuación y no saben" (Comunicación personal, 13 de noviembre de 2012). Esto lleva como consecuencia que los estudiantes no posean un conocimiento previo sólido que garantice el éxito en los nuevos contenidos, y citan la existencia de vacíos en temas como ecuaciones y fórmulas notables. Las dos docentes entrevistadas coinciden en señalar que el rendimiento en matemáticas es pésimo y lo califican como una completa pesadilla; los alumnos estudian solo para pasar y desean que todo sea muy mecánico, por memoria, dejando de lado el razonamiento.

Según la imagen de lo que es un buen profesor de matemáticas, indican que un docente de la materia debe despertar el interés de los estudiantes en la disciplina, escuchar y motivarlos, saber explicar e incentivar el trabajar duro para aprender. Sin embargo, una docente señala la diferencia entre lo que debería ser y lo que actualmente sucede en el aula; aunque indica la importancia de un profesor guía del proceso de enseñanza y aprendizaje, apunta que la tendencia hacia la clase magistral, tradicional, es lo más común. Lo anterior favorecido por la presión de cumplir con el programa de estudios y abarcar la materia suficiente para los períodos de evaluaciones establecidos.

Con respecto a la percepción que los docentes consultados poseen de los profesores de matemáticas, en general, ellos concuerdan que estos sí influyen en la opinión de los estudiantes sobre la materia, que cometen errores y los enmiendan y que aconsejan y enseñan a sus alumnos a estudiar. Además, rechazan que se preocupen solamente por los más aventajados y que no les interesa que el dicente entienda.

Una docente indica que la reprobación en matemáticas es muy alta y que aunque muchos intentan realizar bien su trabajo, a veces el mismo medio ejerce presión en ellos y algunos terminan por adoptar prácticas en donde se busca, no la calidad, sino la mayor aprobación. Ella indica, por ejemplo, "en este momento un montón de aplazados que tengo y hasta cierto punto una presión (...) porque les ayude, que pobrecitos (...)". (Comunicación personal, 13 de noviembre de 2012)

Esta presión por lograr una mayor aprobación lleva a algunos docentes, según la opinión de la profesora, a realizar ejercicios de repaso muy similares a los del examen o a indicarles, antes de la evaluación, en cuáles temas o ejercicios debían concentrarse más.

Dada la importancia que los estudiantes dieron al trato del docente, en general, se profundizó en este aspecto desde la perspectiva de los profesores. Al respecto, una docente apunta que este es un factor que influye en la disposición hacia la materia. La grosería, contestar de mala manera, enojarse al ser cuestionado, reprender ante todo el grupo, 
suponer que algunos temas son básicos y no explicarlos son actitudes que afectan en forma negativa y crea una cultura de miedo hacia los profesores de matemáticas, por lo que los califican como autoritarios, amargados, "antisociales", entre otros.

En este sentido ella manifiesta:

(...) hay profesores muy groseros, muy creídos en Matemáticas, que creen que son superiores entonces eso hace que el estudiante también sienta una limitante ahí para comunicarse adecuadamente. También hay irresponsables que se basan en un libro y les ponen el número de página y vean a ver qué hacen. Entonces eso hace que el estudiante se desmotive. Yo creo que sí, un profesor marca. No es definitivo pero sí marca que un estudiante le guste o no le guste, le cueste o no le cueste una materia. (Comunicación personal, 15 de noviembre de 2012)

Las docentes entrevistadas apuntan que el trato al estudiante es importante y se debe incentivar el desarrollo de valores, dar consejos y estar atento para ver si se detecta que un alumno está en problemas, debe haber un margen de distancia por respeto, debido a que los docentes son una autoridad en el aula. El temor a perder dicha autoridad tiene como consecuencia que los profesores asuman posiciones extremas, de distanciamiento, lo que resulta "más seguro" para ellos.

\section{Comparación de los resultados con estudiantes y docentes}

Al analizar las respuestas dadas por el estudiantado y el grupo docente, es posible observar coincidencias en algunas de ellas, pero también divergencias. Aunque los profesores indican que el reto que representa estar en una clase estimula el desarrollo académico de todos los alumnos, estos no expresan lo mismo.

Con respecto a las creencias asociadas con las matemáticas, ambos grupos coinciden en señalar que los conocimientos de esta disciplina se emplean en otras ciencias, son importantes por su utilidad en la vida cotidiana, que existen diferentes formas de encontrar la solución a un problema, están en continua expansión, muchas cosas quedan aún por descubrir y que el error es un elemento importante en el estudio de la materia. La visión de resolución de problemas es la visión de las matemáticas predominante para ambos, aunque también se destacan características de la visión instrumentalista; lo anterior al considerar que las matemáticas constituyen una acumulación de hechos, reglas y habilidades que pueden ser usadas en la ejecución de algún fin externo. 
En relación con la visión de las Matemáticas, aunque el estudiantado señala, en su mayoría, que estas se basan en manipular números y símbolos, los docentes no destacaron esta característica sobre la disciplina. Coinciden en señalar que las matemáticas son difíciles, importantes, necesarias, que enseñan a pensar y que poseen muchas aplicaciones en la vida cotidiana. Sobre este último punto, ambos grupos de participantes convergen al indicar que la su aplicabilidad es un aspecto muy importante para su enseñanza y que ha estado ausente en las lecciones de dicha materia. Aunque los docentes reconocen que las aplicaciones en el aula se reducen a aspectos muy básicos, dicen que aún falta mucho para lograr crear conciencia sobre la importancia y aplicabilidad de la disciplina. Los alumnos, al respecto, indican que es evidente el vacío existente en la metodología empleada relacionado con este aspecto, pues para muchos de los contenidos matemáticos la pregunta que ellos hacen es para qué sirve.

Relacionado con las matemáticas como disciplina, ambos grupos de participantes señalan que estas pueden ser estudiadas por cualquier persona siempre que muestre dedicación, esfuerzo y hábitos de estudio. La dificultad o no que se pueda tener dependerá de las habilidades de cada estudiante, lo que conllevará más o menos dedicación, así como concentración en clase, pero que esta no constituye, ni debe ser, un obstáculo. Los docentes agregan, además, la importancia de que el estudiantado confíe en sí mismo y mantenga una actitud positiva hacia la disciplina.

Tanto estudiantes como docentes concuerdan en señalar que la enseñanza de las matemáticas se caracteriza por ser mecanicista. Los primeros indican que los profesores siguen una metodología tradicional caracterizada por explicaciones teóricas, ejemplos y ejercicios, enfatizando en procedimientos y reglas; y los segundos indican que los alumnos son quienes, cada vez más, demandan una enseñanza más algorítmica, tipo receta, en la que la comprensión, el razonamiento y el aprendizaje no son la prioridad. Lo requerido por ellos es un "método" aplicable a todos los casos y que sea efectivo para obtener la respuesta correcta.

Ambos grupos de participantes coinciden en señalar que el grado de esfuerzo está asociado al rendimiento en la materia y que para tener éxito en ella se debe, además, tener actitud positiva, controlar los nervios y escuchar con atención en las lecciones. Manifiestan, también, aspectos propiamente ligados al estudiante que afectan su rendimiento en la disciplina, como la vagancia, poco esfuerzo y estudio, entre otras, así como la memoria a corto plazo, lo que implica un "aprendizaje" únicamente para el examen. 
Aunque los estudiantes caracterizan las matemáticas como un obstáculo, señalan que la práctica constante es un aspecto fundamental en el estudio de la disciplina. Por un lado, los docentes, por su parte, abogaron más por el esfuerzo, estudio constante, entendimiento y no memorización y dar prioridad tanto al proceso como al resultado final.

Por otro lado, los docentes enfatizaron en la importancia de que el estudiantado tenga una actitud positiva hacia la materia, aunque la mostrada por estos últimos se calificó como negativa moderada. Esto coincidió con lo señalado por los profesores a nivel general, pues ellos perciben que esta es muy negativa y se refleja, según su opinión, en el conformismo, pereza e indiferencia mostrada por los alumnos. Son pocos los casos de alumnos que poseen un comportamiento distinto.

Asociado a lo anterior, la visión de estudiantes y docentes difiere en cuanto a la importancia del estudio de la disciplina. Aunque los profesores consideran que es primordial en la formación de toda persona, los alumnos apoyan la idea que su estudio debe estar limitado para aquellos que van a continuar carreras universitarias relacionadas con las matemáticas.

Ambos grupos de participantes coinciden en señalar que alrededor de las matemáticas se ha creado una imagen muy negativa, como una asignatura muy difícil con altos índices de reprobación. Sin embargo, son consecuentes al indicar que no existe un solo "culpable" de ello, pues tanto el docente como el estudiante son corresponsables. Aunado a lo anterior, indican que el efecto de factores externos como los medios de comunicación, problemas familiares, sociales y económicos, y la influencia de grupos cercanos a él como los padres de familia, docentes de otras materias y los mismos compañeros, contribuyen a empeorar esta situación.

Relacionado con la imagen de un buen docente de matemáticas, estudiantes y docentes coinciden en que es aquel que sabe explicar, escucha y alienta a los alumnos, despierta su interés e incentiva a dar el mayor esfuerzo en el aprendizaje de la disciplina. Aunque los alumnos indican que los docentes de la materia deberían realizar en clase un "ejercicio de cada tipo", estos no están de acuerdo con dicha afirmación.

Es interesante observar que, con respecto a la tendencia didáctica del profesor, la tradicionalista fue con la que los docentes menos se identificaron, aunque para el estudiantado, y según la observación realizada, esta es la que impera en las lecciones de matemáticas.

Se destaca el hecho de que a los profesores de matemáticas, en general, les interesa, además de la respuesta final, el procedimiento por medio del cual el estudiantado llega a 
esta, atienden dudas, abogan por el entendimiento de la materia y enfatizan su enseñanza en incentivar diversas formas de abordar un problema y no tanto un procedimiento algorítmico. Sin embargo, según la opinión de los alumnos, la comprensión de la materia se basa en adquirir las reglas y procedimientos que los docentes enseñan.

\section{Discusión y conclusiones}

En general, aunque la mayoría de estudiantes señala tener dificultades en la disciplina, destaca que estas se deben a la naturaleza de las matemáticas (subrayan su dificultad), al profesor (falta de compromiso y explicaciones insuficientes) y a ellos mismos (poca atención, compromiso, distracciones). Para el alumnado las explicaciones del profesor juegan un papel trascendental en su aprendizaje, motivación, atención y gusto por la materia. Parte de su culpabilidad se debe a la desmotivación que siente hacia el estudio de las matemáticas, falta de compromiso, deficientes hábitos de estudio, no prestar la atención requerida, entre otros.

Los dicentes califican las matemáticas como una disciplina complicada, confusa, poco interesante, mecánica, aburrida y como una asignatura distinta a las otras materias. Las asocian con exámenes, bajos resultados, rigidez, exactitud, un obstáculo para el logro de los objetivos académicos y, para algunos, les resultan intimidantes, generan temor, inseguridad y bloqueo; propiciados, muchas veces, por el ambiente negativo que se ha creado alrededor de ella.

Esto ha provocado que muchos alumnos asuman el estudio de la disciplina con temor y vean los bajos rendimientos en ella como algo normal. Asociado a lo anterior, la mayoría expresa no querer estudiar más matemáticas e indica que esto deberían de hacerlo, únicamente, los que van a estudiar carreras relacionadas con ellas. Sin embargo, también destaca algunas características positivas como que enseñan a pensar, son valiosas, necesarias, proveen conocimientos a otras áreas y se pueden aplicar en la vida diaria.

Tanto estudiantes como docentes coinciden en señalar que para aprender matemáticas se requiere esfuerzo, dedicación y sentir gusto por la materia; se le da una especial importancia a la ejercitación como el medio por el cual los alumnos adquieren los algoritmos y se instruyen en los procedimientos que deben seguir para obtener la respuesta a los problemas propuestos por el docente. Destacan que los ejercicios en matemáticas poseen una única respuesta correcta, aunque reconocen que hay distintas formas de encontrarlas. Sin embargo, los estudiantes expresan su disconformidad al señalar que, aunque realicen muchos siempre habrá nuevos, que representan retos distintos, por lo que 
aplicar un procedimiento único es imposible. Esto es señalado por los alumnos como una "debilidad" de la materia que, a su vez, es parte de su dificultad. Se recalca que en matemáticas el error se constituye en una fuente de aprendizaje, que permite, cuando se recibe la retroalimentación correspondiente, solventar las deficiencias.

Aunque los estudiantes valoran a las matemáticas como una disciplina útil, no todos se muestran interesados en su estudio. Lo anterior posiblemente se debe a que dan una opinión cuando se refieren a las matemáticas como disciplina y campo del saber y otra cuando las describen como materia escolar. No se visualiza un vínculo entre una y otra; es como si fueran distintas.

Las matemáticas, como disciplina, son calificadas como importantes, necesarias, aplicables y que aportan conocimientos a otras áreas. Como asignatura escolar, es descrita como complicada, confusa, mecánica y aburrida. Desde este punto de vista, las matemáticas en secundaria se deberían limitar, según los educandos, al manejo de conocimientos básicos sin profundizar en contenidos que no tendrían aplicación en una carrera universitaria y desarrollo laboral. Por lo tanto, para la mayoría de ellos, esta asignatura constituye solo un requisito académico. Esto representa un indicio de que existe una brecha entre estas dos visiones y que podría ser consecuencia de la forma en que se ha abordado en el aula.

Para los estudiantes, la motivación y la confianza en sí mismos al enfrentarse con la materia representan elementos fundamentales para el éxito en las matemáticas. La motivación que ellos puedan sentir hacia la materia y su estudio está directamente asociado con su grado de comprensión y su rendimiento en ella. Aunque la mayoría de los estudiantes señala que realizan un gran esfuerzo en la materia, para muchos de ellos esto no se ve reflejado en los resultados. Este hecho ha traído consigo que algunos alumnos se valoren a sí mismos como poco hábiles y consideren que, sin importar lo que hagan, nunca podrán obtener un buen rendimiento en la materia, lo que genera frustración y hastío hacia ella.

El gusto que muchos de los estudiantes sienten por las matemáticas se asocia con su rendimiento académico. Si las notas obtenidas por ellos en las actividades de evaluación son altas, se refuerza su confianza en sí mismos con respecto a su desenvolvimiento en la materia y se motivan en su estudio. En caso contrario, la disposición hacia esta es poco favorable y se genera tristeza, frustración, presión y una serie de pensamiento negativos.

Con respecto a la metodología que los docentes utilizan en las lecciones, los dicentes señalan que esta se caracteriza por sus explicaciones reiterativas, exposición de ejemplos y prácticas iterativas. Una de las críticas que hacen ellos al docente es que, por lo general, los 
ejercicios realizados en clase son de distinta dificultad a los incluidos en las prácticas y los exámenes, por lo que no están desarrollando estrategias para enfrentar estos con éxito.

Aunque los contenidos se desarrollan de forma descontextualizada, ajenos a la realidad del estudiante y donde la aplicabilidad de las matemáticas está ausente, tanto docentes como alumnos reconocen que esta característica es un valor agregado que tiene el aprendizaje de la disciplina, el cual no se está aprovechando. Indican que la forma en que se enseñan las matemáticas no les resulta atrayente.

A pesar de que los docentes tratan de hacer una clase participativa, incluir algunas actividades diferentes y realizar preguntas a los estudiantes, estos no responden como se espera y se muestran desinteresados e indiferentes, por lo que la clase se limita a enseñar procedimientos y reglas. El profesorado indica que prefiere abordar un enfoque tradicional de enseñanza ante la posibilidad de una decepción al emplear distintas estrategias metodológicas.

Es importante señalar, con respecto al punto anterior, dos cuestiones contradictorias entre estudiantes y docentes. Los primeros señalan que las clases de los segundos son rutinarias, poco motivantes y ausentes de actividades innovadoras, orientadas en una tendencia didáctica tradicionalista y enfatizando en el aprendizaje de algoritmos. Los profesores, por su parte, señalan que ellos tratan de no hacer la clase tradicionalista, que incluyen otros aspectos, y que cuando han tratado de realizar una actividad distinta han sufrido una frustración al ver la actitud de los estudiantes, quienes lo que esperan es una receta.

La disposición del profesor, según los estudiantes, constituye un aspecto que influye en su actitud hacia la materia. Un docente que sepa escuchar, aclarar dudas, interactúe con ellos, los motive y genere confianza son algunos de los elementos importantes que ellos señalan. El profesor ajeno a la realidad del estudiante, que no muestra compromiso con el aprendizaje del alumno, que imparte la clase sin hacerlo partícipe, es catalogado como un factor que afecta, negativamente, en los discentes.

La necesidad de que los docentes aborden la parte afectiva y emocional de los estudiantes hacia la disciplina resulta trascendental a partir de los resultados obtenidos. Abordar la relación entre las creencias y actitudes hacia las matemáticas y su efecto en el aprendizaje de la materia puede ser un medio para mejorar la visión de esta y su rendimiento. Cómo el estudiante se perciba a sí mismo como aprendiz de matemáticas determina parte de su éxito o fracaso. Aunado a lo anterior, la visión que poseen de la 
disciplina influye en el por qué y para qué debe estudiarse. Aspectos que no han sido atendidos en la enseñanza actual.

Es en la labor de aula donde el profesor de matemáticas debe considerar la realidad del estudiante, lo que siente, piensa, lo que creé y cómo actúa. No puede ser indiferente a la realidad de sus alumnos. Debe incentivar el estudio de la disciplina y hacer sentir a sus educandos competentes en ella. Debe ser consciente de que existe una brecha entre lo que los estudiantes creen que son las matemáticas como disciplina y que lo perciben de las matemáticas en el colegio. Esto, claro está, no es solo una responsabilidad del profesor, sino de todo el sistema educativo, el cual debe revisarse, actualizarse e implementar innovaciones. No es solamente lo que el estudiante crea de sí lo que ayuda o perjudica. Cómo el docente pueda potenciar y atender las capacidades y necesidades individuales contribuye con este aspecto. La tarea es, entonces, concientizar al docente para abordar estas cuestiones desde su labor diaria y en beneficio de sus propios educandos. 


\section{Referencias}

Abraham, Graciela, Mena, Analía, Rodríguez, M. R., Golbach, M., Rodríguez, M., Galindo, Graciela. (2010). ¿La actitud hacia la matemática influye en el rendimiento académico? En P. Lestón (Ed.), Acta Latinoamericana de Matemática Educativa, 23 (pp. 75-84). México, DF: Colegio Mexicano de Matemática Educativa A. C. y Comité Latinoamericano de Matemática Educativa A. C. Recuperado de http://funes.uniandes.edu.co/4526/

Akay, Hairy y Boz, Nihat. (2010). The Effect of Problem Posing Oriented Analyses-II Course on the Attitudes toward Mathematics and Mathematics Self-Efficacy of Elementary Prospective Mathematics Teachers. Australian Journal of Teacher Education, 35(1), 175. Recuperado de http://ro.ecu.edu.au/cgi/viewcontent.cgi?article $=1329 \&$ context=ajte

Álvarez, Yadira. (2007). Actitudes hacia las matemáticas de los estudiantes de ingeniería de las universidades venezolanas (Tesis doctoral). Departamento de Psicología y Metodología de las ciencias del comportamiento. Facultad de Psicología. Universidad de Málaga, España.

Briley, Jason, Thompson, Tony y Iran-Nejad, Asghar. (2009). Mathematical Beliefs, SelfRegulation, and Achievement by University Students in Remedial Mathematics Courses. Research in the schools, 16(2), 15-28.

Candia, Patricia. (Setiembre, 2009). Actitud hacia las matemáticas en alumnos de ingeniería de tercero y quinto semestres del ITESCA. En Consejo Mexicano de Investigación Educativa (Comp.), Ponencia: X Congreso Nacional de Investigación Educativa. Área 5: Educación y Conocimientos Disciplinares. Recuperado de http://www.comie.org.mx/congreso/memoriaelectronica/v10/pdf/area tematica 05/pone ncias/0310-F.pdf

Contreras, Luis. (1998). Marco teórico sobre concepciones acerca de la enseñanza y el aprendizaje de la matemática (Capítulo 2. Tesis doctoral). Universidad de Huelva, España. Recuperado de http://www.uhu.es/luis.contreras/tesistexto/cap2.htm

Contreras, Luis. (2009). Concepciones, creencias y conocimiento: Referentes de la práctica profesional. Revista Electrónica Iberoamericana de Educación en Ciencias y Tecnología, 1(1), 11-36. Recuperado de http://www.exactas.unca.edu.ar/riecyt/VOL\%201\%20NUM\%201/Doc\%20RIECyT\%2011.pdf

Eleftherios, Kapetanas y Theodosios, Zachariades. (2007). Students' beliefs and attitudes concerning mathematics and their effect on mathematical ability. En D. Pitta-Pantazi y 
G. Philippou (Eds.), Proceedings of the Fifth Congress of the European Society for Research in Mathematics Education, 258-267. Recuperado de http://ermeweb.free.fr/CERME5b/WG2.pdf

Ernest, Paul. (1989). The Impact of Beliefs on the Teaching of Mathematics. En P. Ernest (Ed.), Mathematics Teaching: The State of the Art, 249-254. London: Falmer Press. Recuperado de http://webdoc.sub.gwdg.de/edoc/e/pome/impact.htm

Gil, Nuria, Blanco, Lorenzo y Guerrero, Eloísa. (2005). El dominio afectivo en el aprendizaje de las Matemáticas. Una revisión de sus descriptores básicos. Revista Iberoamericana de Educación Matemática, (2), 15-32. Recuperado de http://www.fisem.org/www/union/revistas/2005/2/Union 002 004.pdf

Gil, Nuria, Blanco, Lorenzo y Guerrero, Eloísa. (2006). El papel de la afectividad en la resolución de problemas matemáticos. Revista de Educación, 340, 551-569. Recuperado de http://www.revistaeducacion.mec.es/re340/re340 20.pdf

Gómez-Chacón, Inés. (2007). Sistema de creencias sobre las matemáticas en alumnos de secundaria. Revista Complutense de Educación, 18(2), 125-143.

Hassi, Marja-Liisa y Laursen, Sandra. (2009). Studying Undergraduate Mathematics: Exploring Students' Beliefs, Experiences and Gains. En S.L. Swars, D.W. Stinson y S. Lemons-Smith (Eds.), Proceedings of the 31st Annual Meeting of the North American Chapter of the International Group for the Psychology of Mathematics Education (PMENA). 5, 113-121. Atlanta, GA: Georgia State University.

Hekimoglu, Serkan y Kittrell, Emily. (2010). Challenging Students' Beliefs about Mathematics: The Use of Documentary to Alter Perceptions of Efficacy. Problems, Resources, and Issues in Mathematics Undergraduate Studies, 20(4), 299-331. Recuperado de http://pdfserve.informaworld.com/233589 921795932.pdf

Martínez, Oswaldo. (2008). Actitudes hacia la matemática. Sapiens, 9(1), 237-256.

Naranjo, Claudia y Segura, Mónica. (marzo, 2010). Representaciones sociales de los estudiantes de la media vocacional sobre las matemáticas y la química. En VI Congreso Internacional Didácticas de las Ciencias y XI Taller Internacional sobre la Enseñanza de la Física. Simposio realizado en la reunión de El Ministerio de Educación de la República de Cuba, La Habana.

Naranjo, María. (2010). Factores que favorecen el desarrollo de una actitud positiva hacia las actividades académicas. Educación. 34, 31-53. 
Op't Eynde, Peter, De Corte, Erik y Verschaffel, Lievan. (2002). Framing students' mathematics related beliefs: A quest for conceptual clarity and a comprehensive categorization. En G. Leder, E. Pehkonen, y G. Törner (Eds.), Beliefs: A hidden variable in mathematics education? (pp. 13-38). Dordrecht, The Netherlands: Kluwer Academic Publishers.

Parra, Hugo. (2005). Creencias matemáticas y la relación entre actores del contexto. Revista Latinoamericana de Investigación en Matemática Educativa. 8(1), 69-90. Recuperado de https://dialnet.unirioja.es/servlet/articulo?codigo=2096245

Sánchez, José, Becerra, Julieta, García, Julieta, Contreras, María. (2010). La dimensión afectiva y el rendimiento en estadística en estudiantes universitarios. En P. Lestón (Ed.), Acta Latinoamericana de Matemática Educativa, 23. México, DF: Colegio Mexicano de Matemática Educativa A. C. y Comité Latinoamericano de Matemática Educativa A. C. Recuperado de http://funes.uniandes.edu.co/4599/

Solé, Isabel. (1999). Disponibilidad para el aprendizaje y sentido del aprendizaje. En C. Coll et. al. (Eds.), El Constructivismo en el Aula (pp. 25-46). España: Editorial Graó.

Stramel, Janet. (2010). A naturalistic inquiry into the attitudes toward mathematics and mathematics self-efficacy beliefs of middle school students (Tesis de Maestría). Disponible en la base de datos de ProQuest Dissertations and Theses. (AAT 3419596)

Suthar, Velo y Tarmizi, Rohani. (2010). Effects of Students' Beliefs on Mathematics and Achievement of University Students: Regression Analysis Approach. Journal of Social Sciences. 6(2), 146-152. Recuperado de http://thescipub.com/html/10.3844/jssp.2006.146.152

Vieytes, Susana. (2009). Factores que afectan las actitudes hacia las matemáticas en los estudiantes de primer año del curso MATE 3001 de la Universidad de Puerto Rico en Utuado (Tesis doctoral). Facultad de Educación, Universidad de P Puerto Rico, Puerto Rico 